San Jose State University

SJSU ScholarWorks

Master's Theses

Master's Theses and Graduate Research

1991

\title{
Characterization of intermediate states in the denaturant induced unfolding of Ribonuclease $A$
}

Rupinder Ghuman

San Jose State University

Follow this and additional works at: https://scholarworks.sjsu.edu/etd_theses

\section{Recommended Citation}

Ghuman, Rupinder, "Characterization of intermediate states in the denaturant induced unfolding of Ribonuclease A" (1991). Master's Theses. 194.

DOI: https://doi.org/10.31979/etd.fxx2-yh3n

https://scholarworks.sjsu.edu/etd_theses/194

This Thesis is brought to you for free and open access by the Master's Theses and Graduate Research at SJSU ScholarWorks. It has been accepted for inclusion in Master's Theses by an authorized administrator of SJSU ScholarWorks. For more information, please contact scholarworks@sjsu.edu. 


\section{INFORMATION TO USERS}

This manuscript has been reproduced from the microfilm master. UMI films the text directly from the original or copy submitted. Thus, some thesis and dissertation copies are in typewriter face, while others may be from any type of computer printer.

The quality of this reproduction is dependent upon the quality of the copy submitted. Broken or indistinct print, colored or poor quality illustrations and photographs, print bleedthrough, substandard margins, and improper alignment can adversely affect reproduction.

In the unlikely event that the author did not send UMI a complete manuscript and there are missing pages, these will be noted. Also, if unauthorized copyright material had to be removed, a note will indicate the deletion.

Oversize materials (e.g., maps, drawings, charts) are reproduced by sectioning the original, beginning at the upper left-hand corner and continuing from left to right in equal sections with small overlaps. Each original is also photographed in one exposure and is included in reduced form at the back of the book.

Photographs included in the original manuscript have been reproduced xerographically in this copy. Higher quality 6" x 9" black and white photographic prints are available for any photographs or illustrations appearing in this copy for an additional charge. Contact UMI directly to order.

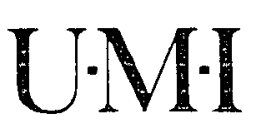

University Microfims International

A Bell \& Howell information Company 

Order Number 1345801

Characterization of intermediate states in the denaturant induced unfolding of ribonuclease $A$

Ghuman, Rupinder Kaur, M.S.

San Jose State University, 1991 



\title{
CHARACTERIZATION OF INTERMEDIATE STATES IN THE DENATURANT INDUCED UNFOLDING OF RIBONUCLEASE A
}

\author{
A Thesis \\ Presented to \\ The Faculty of the Department of Chemistry \\ San Jose State University
}

In Partial Fulfiliment

of the Requirements for the Degree

Master of Science

By

Rupinder Ghuman

August, 1991 


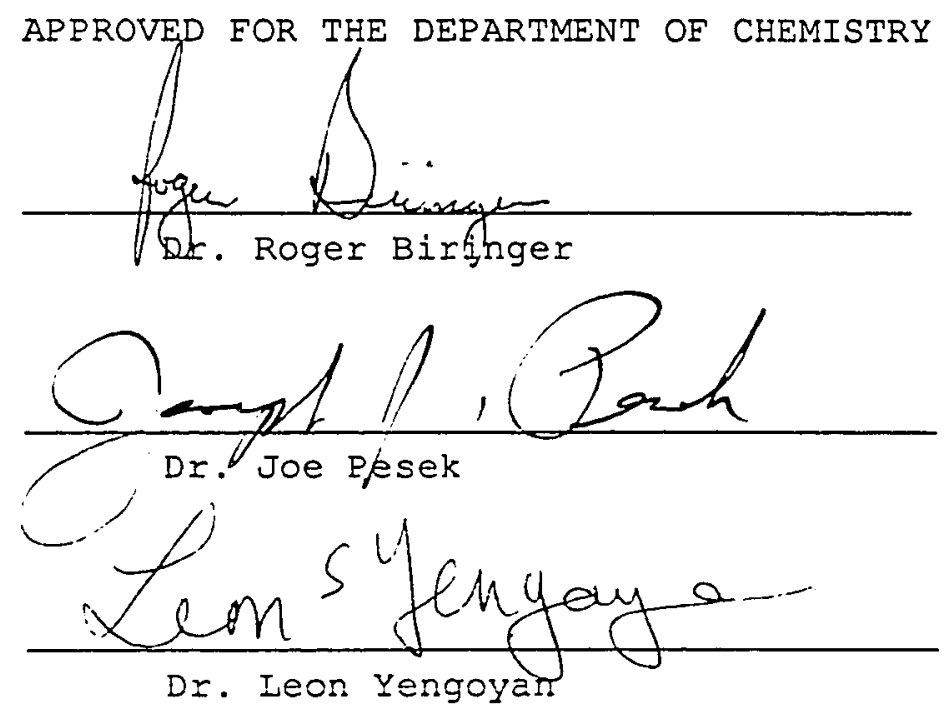

APPROVED FOR THE UNIVERSITY

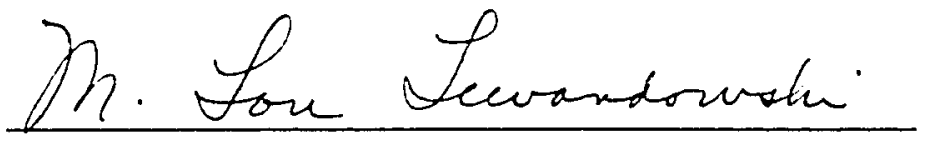




\begin{abstract}
CHARACTERIZATION OF INTERMEDIATE STATES IN THE DENATURANT INDUCED UNFOLDING OF RIBONUCLEASE A
\end{abstract}

by Rupinder K. Ghuman

The focus of the proposed research was to determine the experimental conditions of denaturant concentration which leads to stabilized partially-folded intermediate structures of Ribonuclease $A$ and then to characterize these structures in as precise manner as possible. In addition, denaturants (mostly urea derivatives) with varying abilities to participate in hydrogen-bonding and denaturants which vary in their hydrophobicity were employed to examine the dominant forces in protein folding.

Determination of the conditions which stabilize intermediate states and characterization of these structures was done by using the following structural probes: absorbance, fluorescence, circular dichroism, gradient-gel electrophoresis, and hydrophobic dye binding.

It was observed with absorbance, fluorescence, and circular dichroism that urea and urea derivative denaturants resulted in loss of cooperativity indicating the presence of some intermediate states. Studies with ANS binding revealed that at low concentrations of the denaturant, proteins undergo small conformational changes. 


\section{ACKNOWLEDGEMENTS}

I am greatly indebted to my research advisor, Dr. Roger Biringer. It was his inspiration, guidance, and support which enabled me to complete my graduate work. From him, I shall always take with me the following lessons: to think scientifically, to question, and to be creative.

I would also like to thank Dr. Joseph Pesek and Dr. Ieon Yengoyn for their valuable contributions to my thesis.

No less important were the contributions made by those who provided the very important support and encouragement throughout my school career: my parents, who have always shown pride in me and made me feel that I could accomplish anything that $I$ set my mind to do; my biothers, for their moral support and help; my husband, who made it possible for me to complete the thesis; and my fellow classmates and the San Jose State University Chemistry Department in general whose help, friendship and understanding made the following work possible. 
TABLE OF CONTENTS

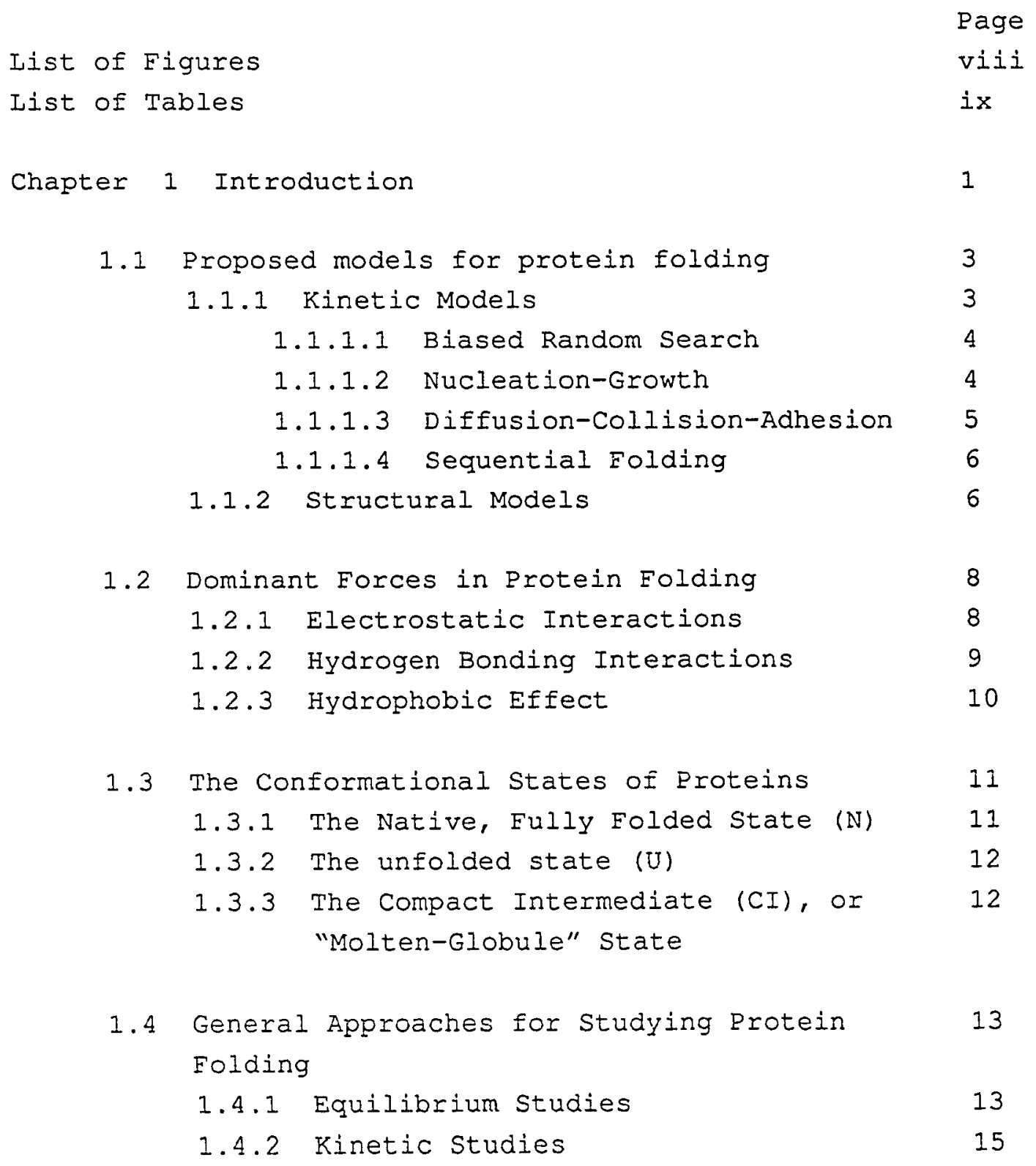


1.5 Detection and Characterization of 16

Intermediates along the Denaturant Induced

Equilibrium Unfolding Pathway

1.5.1 Effects of Different Denaturants on 18

the Conformational Stability of

Ribonuclease $A$

1.5.2 Ribonuclease A 19

1.5 .3 Spectroscopic Probes Used to Monitor 19 Protein Unfolding

1.5.3.1 Absorbance 20

1.5.3.2 Fluorescence 20

1.5.3.3 Gradient gel electrophoresis 21

1.5.3.4 Circular Dichroism 22

1.5.3.5 Hydrophobic dye binding 24

Chapter 2 Materials and Methods 26

2.1 Materials 26

2.2 Methods 26

2.2.1 Purification of Ribonuclease A 26

2.2.2 Preparation of Carboxymethylated 26

Ribonuclease A (CM-RNase A)

2.2.3 Absorbance and Fluorescence 27

Measurements

2.2.4 Unfolding Monitored by Circular 28

Dichroism

2.2.5 Gradient-Gel Electrophoresis 28

2.2.6 Hydrophobic Dye Binding Measurements 31

2.2.7 Denaturation Curve Analysis 31

2.2.8 The Analysis of ANS Binding Curve 34

Chapter 3 Results and Discussion 35

3.1 Absorbance and Fluorescence 35

vi 
3.1.1 Effect of Denaturants on Absorbance and Eluorescence

3.2 Circular Dichroism 41

3.2.1 Effect of Denaturants on Circular 41

Dichroism

3.3 Hydrophobic dye Binding $\quad 44$

3.3.1 Effect of Denaturants on Hydrophobic 46 dye binding

3.4 Denaturant-gradient gel electrophoresis 49

3.4.1 Effect of Denaturants on the Gradient 49 gel electrophoresis

3.5 Comparison of the Data Obtained from 54 Different Spectroscopic Techniques

3.6 Conclusion $\quad 58$

References $\quad 62$

vii 
1. Greek Key Structure 7

2. Equilibrium Transitions 14

3. The $C D$ for various structures 23

4. Denaturation Curve Analysis 33

5. Absorbance and Flu. Denaturation Curve 36

6. Fu vs M Ethyl urea 38

7. $\Delta G$ vs M Ethyl Urea 38

8. Fu vs M denaturants-Fluorescence 39

9. -Slope vs Cm- Fluorescence 42

10. Fu vs $M$ denaturants- Circular Dichroism 43

11. Cm Fluorescence vs Cm Circular Dichroism 45

12. Relative Fluorescence Vs M Denaturants 47

13. DMSO Gradient gel 50

14. Urea Gradient gel 51

15. Rf VS M DMSO 52

16. Rf vs M Urea 52

17. Fu vs $M$ denaturant-gels and CD 53

18. Eu vs M Ethyl Urea 55

19. Fu vs M GuHCl 57

20. Fu VS M DMSO 59 


\section{LIST OF TABLES}

Table

Page

1. $\mathrm{Cm}$ and $\Delta \mathrm{G}^{\mathrm{aO}}$ values for Fluorescence and 40 Circular dichroism

2. Transition midpoints for transition 1 (ANS 1) 46 and Transition 2 (ANS 2) for all the denaturants 


\section{Chapter 1: INTRODUCTION}

The mechanism by which a protein attains its tertiary structure is not fully understood. This problem has been a major challenge in biophysical chemistry. The fact that proteins with a given amino acid sequence always attain the same native structure, and different sequences lead to different structures indicates that the amino acid sequence "codes" for native structure.

Knowledge of the "code" which determines the protein folding pathway would be an invaluable tool in medical, pharmaceutical and scientific research. Furthermore, with the knowledge of the folding code, it will be possible to design and tailor proteins with specific properties using recombinant DNA techniques.

The ability to design de novo proteins requires an understanding of how sequences control folding. The problem of designing an amino acid sequence to adopt a desired threedimensional structure has been addressed recently: Regan and DeGrado (1988) have designed and synthesized a novel protein that folds into a remarkably stable conformation; Richardson and Richardson (1990) also have designed a four-helix cluster protein known as felix. Remarkable advances like these and recent progress in genetic engineering may, one day, enable one to design a protein with a particular structure and function.

In addition, this knowledge would also provide insight with regard to protein stability and thus allow for the engineering of proteins which are more stable than their naturally occurring counterparts. The increased stability of proteins would provide a longer shelf life and thus would be a great advantage for pharmaceutical corporations.

The elucidation of the mechanism of the folding pathway of globular proteins has been the subject of active research. 
The general hope is that determining the folding pathway of small monomeric proteins will shed some light on the general principles which underlie the protein folding process. Current interest in the mechanisms and pathways of protein folding centers on the nature of intermediate states. In order to elucidate the folding mechanism of a particular protein it is necessary to characterize these intermediates and place them in the correct order on the folding pathway.

The problem of determining the folding pathway of a protein, and of understanding the folding mechanism in terms of the energetics and structures of the intermediates has its origin in work that goes back at least one half century. Realization of the significance of these early observations developed slowly, due primarily to the limited knowledge of protein structure. In 1929, wu concluded that protein denaturation was a process involving the unraveling of the polypeptide chain. He also proposed that native proteins involve regular repeated patterns of folding of the polypeptide chain into a three-dimensional network somewhat resembling a crystal and held together by non-covalent linkages. Three years later, Northrop (1932) reported that the thermal denaturation of trypsin at $\mathrm{pH} 2$ follows a reversible transition curve. In early 1950's, Kauzmann and his co-workers were able to demonstrate that protein denaturation is the unfolding of the ordered, threedimensional structure of a protein. In the late 1950's, Christian B. Anfinsen made a remarkable discovery. He demorstrated that the amino acid sequence of a protein, a one-dimensional trait, was fully sufficient to specify the molecules ultimate three-dimensional shape and biological activity.

In 1951, Pauling et al. discovered that proteins contain specific types of structures known as $\alpha$-helix and paraliel and anti-paraliel sheets by using $\mathrm{X}$-ray crystallography. These were first called "secondary structures" by 
Linderstorm-Lang (1952). Not long afterward Kendrew and coworkers (1960) solved the $\mathrm{X}$-ray structure of myogloblin. By the mid 60's, there was enough information at hand to further investigate the pathway and mechanism of folding.

In the last few years, technological advances have made it possible to obtain the precise knowledge of the threedimensional structure of proteins. The x-ray structure of an increasing number of proteins can now be obtained with high resolution, allowing the knowledge of protein structure at the atomic level. The $\mathrm{x}$-ray crystallographic studies provide an important piece of information for understanding how the architecture of a protein molecule could be built from the extended polypeptide chain and thus these studies can contribute significantly to improving the hypothesis concerning the folding pathway.

Since the earlier works, it has been recognized that the folding of many proteins proceed via intermediate controlled process (Matheson \& Scheraga, 1979; Biringer \& Fink, 1982; Schmid \& Baldwin, 1979). The present investigation also reveals the presence of intermediate structures and thus further studies can be done to characterize these intermediate structures.

\subsection{Proposed Models for Protein Folding}

Many models of how proteins might fold have been proposed with varying degrees of detail and predictions that are capable of being tested. Most have stressed roles for secondary structure, since such regular conformations seem most likely to be stable in isolation and at early stages of folding (Baldwin, 1986). At opposite extremes, there are models that predict a single pathway and one that predicts a vast number of pathways. These models are of two kinds: kinetic and structural. Since structural data on folding intermediates are only now starting to be available, both classes of models still consist of guesses about the folding process. Structural models are based chiefly on the success 
of x-ray crystallography and NMR techniques in solving the three-dimensional structure of native proteins.

\subsubsection{Kinetic Models}

The aim of kinetic models is to indicate the dominant intermediates and give the factors that control the rate of folding without being too specific about the structures of the intermediates. Different kinetic models have been proposed by many research groups. The following four kinetic models are most widely accepted.

\subsubsection{Biased Random Search}

The possibility that proteins might fold by a purely random search of all possible conformations was considered by Levinthal (1968) and then dismissed, because the time required for folding would be impossibly long; for example, in the simplest model, such a random search would take on the

order of $10^{50}$ years for a protein consisting of 100 amino acids (Karplus \& Weaver, 1976). However, it is possible that a biased random search could occur in a reasonable time.

With the use of computer-simulated folding for a lattice model, it is possible to show that the number of possible chain conformations is drastically reduced if only selfavoiding (sterically possible) conformations are allowed (Levitt, 1975). Levitt also found that a significant fraction of the self-avoiding conformations are fairly compact, and he suggested that rapid folding begins whenever the unfolded chain assumes a backbone conformation sufficiently like that of the native protein. This result, taken together with the possibility of a rapid and nonspecific collapse when refolding is initiated, suggests that a biased random search could play an important role in early stages of folding (Levitt, 1975). 
1.1.1.2 Nucleation-Growth

The nucleation-rapid-growth mechanism was initialiy considered to be one of the most likely explanations of how folding could occux rapidly. It envisages the formation of a nucleation center by random conformational fluctuations of the unfolded protein that can proceed rapidly to completion. The nucleated molecule is not observabie as a populated species either because folding occurs rapidiy after nucleation or because the nucleus is unstable by itself and breaks down if it is not stabilized by further folding. In addition, its formation would be rate-limiting step in the refolding (Wetlaufer, 1973).

Populated kinetic intermediates have been demonstrated in the folding of several proteins. It appears therefore, in these cases, folding is not a nucleation-limited reaction.

\subsubsection{Diffusion-Collision-Adhesion}

In this microdomain coalescence model, short segments of the unfolded chain fold independently into microdomains. These are unstable, but they diffuse, collide, coalesce, and eventually become stable (Karplus, 1976; Ptitsyn, 1975). Each of the microdomains can be described as being in equilibrium between native-iike structure in a particular region and the unfolded random-coil structure. A stepwise folding by Diffusion-Collision Adhesion mechanism begins with two units coalescing to form a slightly more stable entity which in turn collides with third entity, and so on. The attractive forces that stabilize the coalesced microdomains are short range and probably involve hydrophobic interaction and exclusion of water. Once the segments have diffused together, the balance between hydrophobic attraction and van der Waals' repulsion determines whether or not coalescence can occur in a given collision. This also requires that segments have correct secondary structure.

In contrast to the difficulties that are found with the random-search chain propagation model, available structural 
and other data suggests that the diffusion-collision model can serve as the basis for a mechanism of protein folding.

\subsubsection{Sequential Folding}

The sequential folding pathway occurs in a unique and definite sequence of steps. The simplest model is as follows:

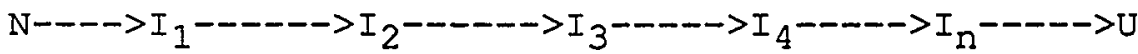

where $N$ and $U$ represent native and unfolded, respectively, and $I_{i}$ are the various intermediate structures. These intermediates would have structures somewhere between native and unfolded states. To demonstrate sequential folding, it is necessary to show that there are specific, well populated intermediates and that their formation and decomposition are coupled in a sequential manner. The presence of such intermediates has been demonstrated in several laboratories (Schmid \& Baldwin, 1979; Biringer \& Fink, 1988; Kim \& Baldwin, 1980).

\section{1 .2 Structural Models}

The aim of the structural models is to give the actual structures of intermediates as well as their order on the pathway, without being too specific about the factors that control the rate of folding. In recent years, the increasing number of $x$-ray crystal structures has stimulated the proposal of many structural models for protein folding. Some models for the folding of an entire class of proteins postulate that folding begins with a "Primitive" hydrogenbonded structure that breaks down to generate the observed structure. The model postulated by Ptitsyn and Finkelstein (1980) for all beta proteins is a long two-stranded antiparallel beta structure with a central hairpin loop (Figure 1A). The Greek key pattern of connections between beta strands then results from breaking this hairpin helix into shorter segments by opening unpaired loops (Eigure 1). 


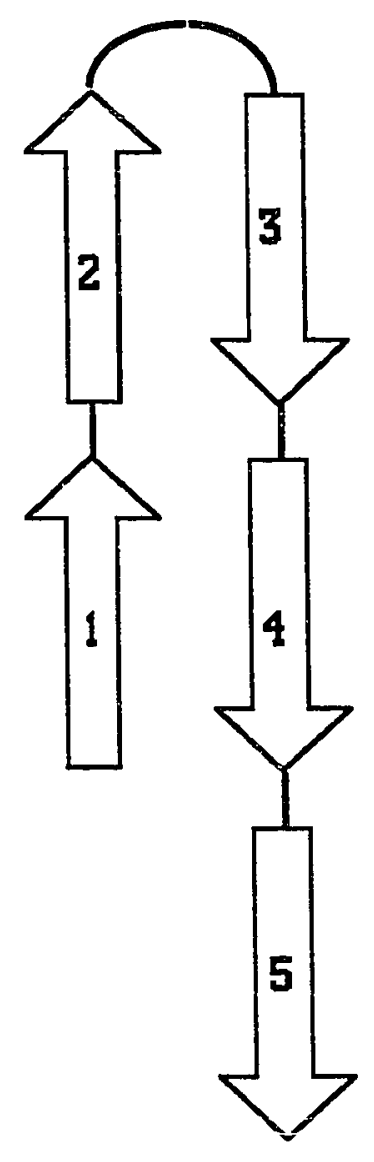

品

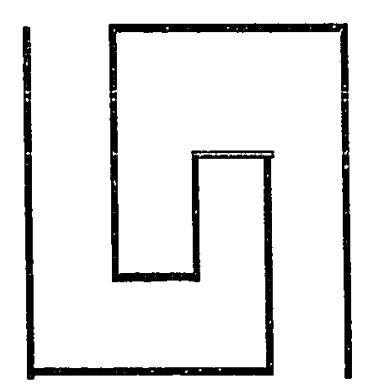

B

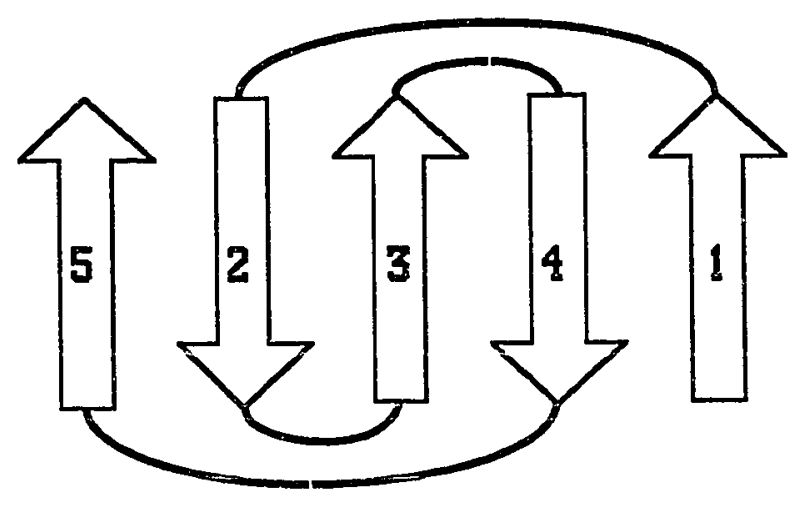

[

Figure 1. Super-secondary structures in proteins. A) Primitive beta-sheet formed in early intermediates, B) Greek-key pattern, C) Greek-key motif of antiparallel sheets formed from primitive beta-sheet. 


\subsection{Dominant Forces in Protein Folding}

Forces, such as electrostatic interactions, hydrophobic interactions, hydrogen bonding and van der wals, contribute to the structural features of globular proteins: their extraordinary compactness, their core of nonpolar residues, and their considerable amounts of internal architecture.

\subsubsection{Electrostatic Interactions}

The dominant folding forces were first assumed to be electrostatic in nature because acids and bases were among the earliest known denaturants. The electrostatic interactions depend on the number and on the distribution of the charges, and, therefore, differ in the folded and unfolded states of a protein conformation. There are two different ways in which electrostatic interactions can affect protein stability:

1. Classical electrostatic interactions are the nonspecific repulsions that arise when a protein is highly charged, for example, at extremes of $\mathrm{pH}$. At extreme $\mathrm{pH}$ values, there are a large number of charges of the same sign; therefore, the repulsions may become strong enough to provoke unfolding of the protein.

2. Specific charge interactions can also affect stability. For example, ion pairing (salt bridging) occurs when oppositely charged amino acid side chains are in close proximity. A recent polyelectrolyte model of proteins (Stigter \& Dill, 1990) shows instead that increasing salt will reduce the electrostatic free energy of the unfolded state of myogloblin more than the folded state. Increased salt shields the charge repulsions in the unfolded molecule more effectively than in the folded molecule at low pH, probably because of better penetration of the salt solution into the unfolded molecule. 


\subsubsection{Hydrogen Bonding Interactions}

A hydrogen bond occurs when a hydrogen atom is shared between two electronegative atoms. A hydrogen bond is primarily a linear arrangement of donor, hydrogen, and acceptor and is comprised of electrostatic, dispersion, charge-transfer, and steric repulsion interactions (Vinogradov \& Iinnell, 1971). The strength of the hydrogen bond ranges between the nonbonded interactions and covalent bonds. The intrinsic energy of hydrogen bonds is usually -2 to $-8 \mathrm{kcal} / \mathrm{mole}$ and the strength decreases as the distance between hydrogen and the acceptor increases.

The hydrogen bond between the peptide carbonyl and amide groups of the backbone is the most frequently observed in proteins. It stabilizes regular structures such as alpa helices, beta structures, and turns. The energy of the peptide hydrogen bond varies as a function of the nitrogenoxygen distance $(R)$ and the hydrogen bond angle $\phi$, with a minmum of $-4.5 \mathrm{kcal} / \mathrm{mole}$ (for $\phi=0^{\circ}$ ). Also, the side chain polar groups form hydrogen bonds with nearby polar groups. Many polar groups are usually located at the outside of the molecule and, therefore, they are able to form hydrogen bonds with neighboring water molecules.

Solvent denaturation studies indicate that hydrogen bonding is not the dominant folding force (Singer, 1962: Edelboch \& Osborne, 1976). If hydrogen bonding was the dominant folding force, then solvents that form strong hydrogen bonds to the peptide backbone should compete effectively with internal hydrogen bonds and thus unfold the protein. Those solvents that do not affect hydrogen bonding should not affect stability significantly. For example, the effectiveness of tetraalkylammonium salts to denature proteins depends on the number of methylene groups, indicative that it is the hydrophobic interaction, rather than hydrogen bonding, which determines stability (Lupu-Lotan et al.,1965). In general, the peptide carbonyl group is a 
strong hydrogen acceptor and the peptide amino group is weak donor. Singer (1962) has pointed out that the solvents useful for competing with the peptide hydrogen bond would be those which are stronger donors than the amide group; the peptide bond will generally compete effectively against solvents that are weaker hydrogen acceptors than the carbonyl group. For example, dioxane is only a hydrogen-bond acceptor and therefore should not denature proteins if hydrogen bonding were the dominant forces (Singer, 1962). However, denaturation of proteins by dioxane suggests that hydrogen bonding is not the dominant force in folding.

\subsubsection{Hydrophobic Effect}

More than 30 years after Kauzmann's (1959) insightful hypothesis, there is now strong evidence that hydrophobicity is the dominant force of protein folding. It now appears that it is the absence of interactions between the nonpolar molecules and water, rather than a favorable interaction between the nonpolar groups themselves, that is a major factor in the stability of protein. This has come to be known as the "hydrophobic effect."

A considerable body of more recent evidence continues to support the view that hydrophobicity is the dominant force of folding. First, spectroscopic and high-resolution differential scanning calorimetry experiments show the resemblance of the temperature dependence of the free energy of folding and the temperature dependence of the free energy of transfer of nonpolar model compounds from water into nonpolar media (Pace, 1975). Second, a large number of crystal structures of proteins have become available which show that a predominant feature of globular protein structures is the sequestered nonpolar residues in a core where they largely avoid contact with water (Chothia, 1976; Wertz \& Scheraga, 1978). Third, protein stability is affected by different salt species in the same rank as the lyotropic series; this is generally taken as empirical evidence for 
hydrophobic interactions (von Hippel \& Schleich, 1969). Fourth, the hydrophobicities of residues in the cores of protein appear to be more strongly conserved and correlated with structure than other types of interactions (Bowie et al., 1990). Fifth, computer simulations of incorrectly folded proteins show that the principal diagnostic of incorrect folding of proteins, apart from inappropriate burial of charge, is the interior/exterior distribution of hydrophobic residues (Baumann et al., 1989).

\subsection{The Conformational States of Proteins}

Different protein conformations differ oniy in the angle of rotation about the bonds of the backbone and the orientation of amino acid side chains. There are three conformational states of proteins which have been examined in great detail: the native state, the unfolded state, and the compact intermediate state.

\subsubsection{The Native, Fully Folded State (N)}

The native conformations of proteins are known in great detail from the structures determined by $x$-ray crystallography and by NMR studies. The native state is considered to be a single conformation with varying degrees of flexibility, but it can be defined as a macro-state made up of a finite number of micro-states with few exceptions. Flexibility is greatest at the protein surface, where some side chains and a few loops have alternative conformations or no particular conformation that is energetically preferred. Flexibility is least in the interior, but even there, sidechain rotation occur. Most tyrosine and phenylalanine sidechain aromatic rings are flipping by $180^{\circ}$ on the millisecond time-scale (Wagner, 1983).

Most of the interactions within the folded state are more favorable energetically, in both enthalpy and free energy, than the corresponding interactions of the unfolded state. These interactions therefore contribute to the net 
stability of the folded state.

\subsubsection{The Unfolded State (0)}

The fully unfolded protein is a random coil, in which the rotation about every chemical bond of the polypeptide is independent of every other bond rotation and determined only by the local stereochemistry of the polypeptide backbone. Steric repulsions are significant between atoms close in the covalent structure, and place limitation on the local flexibility. Because of the large number of possible conformations, the unfolded state is poorly defined.

Experimental proof of the heterogeneity of the unfolded state first became apparent when Garel and Baldwin (1973) found that unfolded ribonuclease $A$, with the disulfides intact, consisted of several populations of molecules which fold to the native state at different rates. Two years later, a possible explanation for the heterogeneity of unfolded states was suggested by Brandts and co-workers (1975). They proposed that the different populations were defined by the isomerization state of proline residues. In the folded state, each such bond is either cis or trans in essentially all of the molecules, whereas, in the unfolded state, there is an equilibrium between two isomers at each such peptide bond. Folding to the native state, therefore, would involve the rate limiting isomerization of proline residues.

\subsubsection{The Compact Intermediate (CI), or "Molten- Globule" State}

Under particular conditions of $\mathrm{pH}$, salt, temperature a variety of proteins exist in stable conformations that are neither fully folded nor fully unfolded. These conformations have sufficient similarities to suggest that they are third stable conformational states (Ptitsyn, 1987). The most common properties of these states as described by Creighton (1990) are:

1. The overall dimensions of the polypeptide chain are 
much less than those of a random coil and only marginally greater than those of fully folded state. 2 . The average content of secondary structure is similar to that of the folded sate.

3. The interior side-chains are in homogeneous surroundings, in contrast to the asymmetric environments, they have in the fully folded state. 4. Many interior amide groups exchange hydrogen atoms with the solvent more rapidly than in the folded state, but more slowly than in the fully unfolded state. 5. The enthalpy of the compact intermediate state is very nearly the same as that of the fully unfolded state, substantially different from that of the native state.

6. Interconversions with the fully unfolded state are rapid and noncooperative, but slow and co-operative with the fully folded state.

\subsection{General Approaches for studying Protein Folding}

Equilibrium transitions and kinetics of unfolding or refolding are two general approaches to study protein folding.

\subsubsection{Equilibrium Studies}

It is well accepted hypothesis that the equilibrium unfolding transitions of many small globular proteins can be represented, to first approximation, as a cooperative twostate transition. Therefore, the two-state approximation is a good working model.

The two-state process is characterized by a single, rather abrupt transition curve (Eigure $2 A$ ) and thus only fully folded $(N)$ and fully unfolded states $(U)$ are observed. At the midpoint of the unfolding transition, 50 percent of the molecules are $\mathrm{N}$ and 50 percent are $\mathrm{U}$, rather than all, or any significant fraction, of the molecules being half-folded. The two-state approximation can only be applied if no 
A

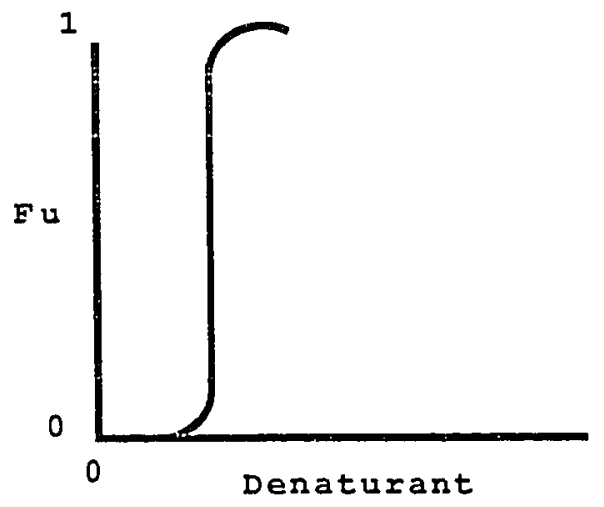

C

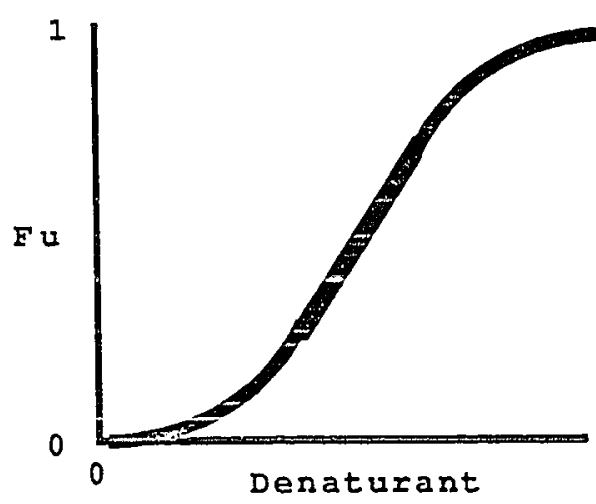

B

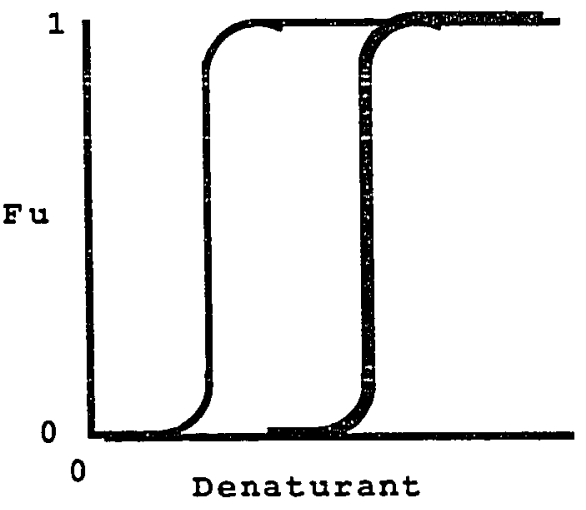

D

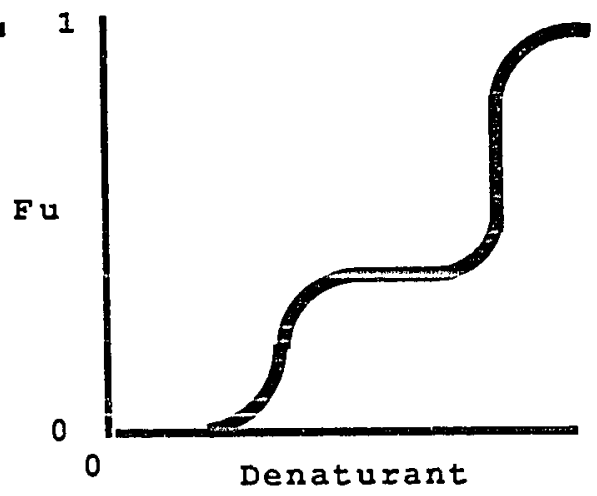

Figure 2. Simulations of equilibrium unfolding plots for various unfolding models. Fu represents the fraction of unfolded molecules (Calculations for Fu data are described in Sec.2.2.7). A) Simple two-state, B) Noncoincident transitions, C) Noncooperative, D) Simple three-state. 
intermediate structures are populated at equilibrium. These studies can then be used to evaluate the differences between the native and unfolded state.

Under certain circumstances, cooperative transitions can yield insight regarding the structure and stabilization of intermediate states. If two spectroscopic probes (e.g., molar ellipticity at $222 \mathrm{~nm}$ and absorbance at $286 \mathrm{~nm}$ ) are employed and each unfolding transition is cooperative, but the transitions are noncoincident (Figure 2B), then a stabilized intermediate state exists under the conditions between such transitions. Examination of these intermediate structures can be very useful in elucidating the mechanism of folding.

If one observes a non-cooperative transition then twostate approximation does not apply (Figure $2 \mathrm{C}$ ). In a noncooperative transition, there exists low levels of intermediates which are nearly indistinguishable from native or unfolded states. As the degree of non-cooperativity increases, the stability of the intermediate structures is increased and they can be observed over longer range of denaturant concentrations.

The unfolding process cannot be described by a two-state approximation when the transition curve displays one, or more than one, well delinated plateau (Figure 2D). In this transition curve, the native state is in equilibrium with the intermediate which is in equilibrium with the unfolded state: the intermediate state being highly populated in the plateau region.

\subsubsection{Kinetic studies}

Kinetic studies are done to examine and characterize the structures of well-populated intermediates. In order to examine kinetic intermediates, the folding transition should be reversible and the native protein should be recovered in $100 \%$ yield upon refolding. In a reversible reaction the folding can be studied from both ends, i.e., in the folding or the unfolding direction. 
If folding can be approximated by a simple two-state transitions then the kinetics of protein unfolding are remarkably simple. In this situation, a single kinetic phase is observed, indicating that all the molecules have the same probability of folding or unfolding and that no partially unfolded intermediates accumulate to substantial levels.

In many cases, multiphasic folding kinetics are observed. The kinetics of refolding to the native conformations are frequently complicated by the effects of the proline peptide bond isomerization, a high energy event, which makes unfolded protein kinetically heterogeneous. Therefore, the folding of each population molecules is characterized by unique rate constants.

Proline isomerization as a slow step in protein folding was suggested by Brandts \& co-workers (1975) as a possible explanation for the two unfolded forms of RNase $A$. RNase $A$ contains both fast-folding (Uf) and slow-folding (Us) forms (Schmidt, 1986). Uf and Us differ in the cis-trans configuration of $\mathrm{X}$-proline peptide bonds: Uf has all essential prolines in the same configuration as in the native state and thus folds rapidly to the native state; Us species contain at least one incorrect proline isomer and thus folding is slower. The proline isomerization is not the only rate limiting event, but others are also possible: for example, disulfide isomerization, the formation of monomers in the case of oligomeric proteins, or slow reshuffling reactions of native-like intermediates.

\subsection{Detection and Characterization of Intermediates along the Denaturant Induced Equilibrium Unfolding Pathway}

The focus of the proposed research is to determine the experimental conditions of denaturant concentration which leads to stabilized partially-folded intermediate structures of ribonuclease $A$ and then to characterize these structures in as precise manner as possible. In addition, denaturants 
with varying abilities to participate in hydrogen bonding and denaturants which vary in their hydrophobicity were employed to examine the dominant forces in protein folding. In our studies, we chose different denaturants (mostly urea derivatives) which were further categorized in the following three categories: Denaturants which differ in their hydrogen bond donor ability, denaturants which differ in their hydrogen bond acceptor ability, and denaturants which differ in their hydrophobic properties.

Determination of the conditions which stabilize intermediate states and characterization of the structures were done by using the following structural probes: absorbance, fluorescence, circular dichroism, gradient gel electrophoresis, and hydrophobic dye binding. The absorbance and fluorescence of the protein was monitored to obtain information regarding the changes in the overall tertiary structure of the protein. Information regarding the changes in the polypeptide backbone conformation was obtained by circular dichroism. Denaturant gradient gel electrophoresis was performed to get an idea about the changes in the overall hydrodynamic radius (overall tertiary structure). Hydrophobic dye binding assays were used to monitor minor structural changes in the protein structure. Studies by these probes also yields additional information regarding the stability of the protein, and intermediate structures.

In order to determine the folding pathway for a protein it is necessary to have structural information regarding each transient state between native and unfolded conformations as well as that for the native and unfolded states and their relative energy states. Analysis of the data by denaturation curves enables one to estimate the conformational stability of the protein. For example, how much more stable the globular native conformation of the protein is in comparison with the unfolded conformation. 


\subsubsection{Effects of Different Denaturants on the Conformational stability of Ribonuclease $A$}

The ribonuclease A unfolding transition induced by denaturant was discovered by Harrington and Schellman (1956) and has since been intensively studied by a number of workers.

The addition of large quantities of denaturants with obvious hydrogen-bonding potential can markedly alter the stability of macromolecular conformations, in general, driving them toward the unfolded form. This effect might be attributed to the ability of these reagents to function, more effectively than water, as competitors for hydrogen-bonding groups normally involved in inter- or intra-chain hydrogen bonding within the molecule. In fact, there is considerable evidence that this is the case. On the other hand, these reagents also seem to have an effect on the stability of hydrophobic interactions.

The action of hydrogen bonding solvents on proteins is a function of its proton donor or proton acceptor character. The energy of hydrogen bonds depends on the nature of the solvent and the competition between intramolecular peptide hydrogen bonds and hydrogen bonds with solvent molecules, $S$,

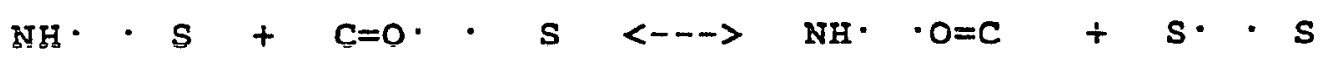

This necessarily depends on the relative strengths of hydrogen bonds between solvent molecules and those between protein and solvent. When the solvent molecules are linked by strong hydrogen bonds, the equilibrium is shifted toward right (i.e., the stabilization of peptide hydrogen bonds).

The denaturant induced unfolding transition was examined for the following denaturants: urea, guanidinium hydrochloride, ethylurea, N-methylurea, 1,3-dimethylurea, formamide, acetamide, and dimethyl sulfoxide. Previous 
studies have revealed that urea and $\mathrm{GuHCl}$ form stronger hydrogen-bonds with the peptide carbonyl and amide groups of proteins whereas solvent such as dimethyl sulfoxide have a very weak tendency to disrupt peptide hydrogen bonds (Singer, 1962). This presumed difference can be used as the basis of a simple test to determine whether the folding is controlled by hydrogen-bonding or hydrophobic interactions. In this study, a hierarchy of denaturants with hydrogen-bonding ability was used. Therefore, these denaturants are suitable as conformational probes in the method of solvent perturbation.

\subsubsection{Ribonuclease A}

The protein chosen for this study was bovine pancreatic ribonuclease $A$ (RNase $A$ ). RNase $A$ is a small single domain protein with 124 amino acid residues. This particular protein offers a number of advantages (Biringer and Fink, 1982): (1) The crystal structure is known; (2) Its folding is characterized by multiple intermediate states; (3) Its solubility in water and other solvents is very high; (4) It has a low tendency to aggregate even in the unfolded state; (5) Its unfolding and subsequent refolding is reversible; and (6) It has been well studied by our group and others.

RNase $A$ offers a number of intrinsic probes of structure. RNase A contains six tyrosines which are equally distributed between the two wings. Three of the tyrosines are relatively exposed in one of the wings and the other three tyrosines are quite buried within the hydrophobic core. These two wings are composed of a bent beta sheet like structure with which three alpha helices are associated. The environment of tyrosine residues can be followed by absorbance and fluorescence.

\subsubsection{Spectroscopic Probes Used to Monitor Protein Unfolding \\ The unfolding of RNase $A$ by different denaturants was monitored by the following spectroscopic probes: absorbance,}


fluorescence, circular dichroism, denaturant-gradient gel electrophoresis, and hydrophobic dye binding.

\subsubsection{Absorbance}

The ultraviolet absorption maxima of proteins undergo shifts to shorter wavelengths (blue-shift) with a slight decrease in intensity when the secondary and tertiary structures of the molecules are disrupted. This phenomena can be used to monitor the unfolding transition of RNase $A$ when exposed to different concentrations of the denaturant. Absorbance of UV light by peptide bonds and non-aromatic amino acid is not very sensitive to conformation or environment, whereas the aromatic rings of phenylalanine, tyrosine, tryptophan residues are.

In case of RNase $A$, the absorbance properties of the six tyrosine residues were monitored. The absorbance properties of a tyrosine residue are dependent on its microsolvent environment. The higher the polarizability of the environment, the lower the wavelength maximum. In case of native RNase $A$, three tyrosines are exposed to the environment and three are buried within the hydrophobic portion (non-polar regions) of the protein. When the RNase A unfolds, the buried tyrosines are exposed to solvent and thus the environment around them changes from a more polarizable environment to a less polarizable environment. This change in the environment will cause a decrease in the energy separation between $\pi$ and $\pi^{*}$ electronic states and cause a red shift in the absorbance spectrum.

\subsubsection{Eluorescence}

The fluorescence quantum yield of aromatic groups is much more sensitive to the local environment than the molar absorption. Therefore, fluorescence is a most effective technique for monitoring conformational changes. The sensitivity of fluorescence is a consequence of the 
relatively long time a molecule stays in an excited singlet state before deexcitation. During the longer lifetime of the excited state, all kinds of processes can occur, including solvent perturbations, local conformational changes, and any processes coupled to translational or rotational motion.

The quantum yield for tyrosines is higher in the unfolded state than the native state. As a protein unfolds with the increasing concentration of denaturant, quenching of the buried tyrosines decreases and hence the quantum yield increases. Tyrosine quenching is due to the partial abstraction of the phenolic proton in the excited state by a proton abstractor such as carbonyl or amide group (Brand \& Bernard, 1967). Abstraction of the proton results in a significantly reduced quantum yield. The increase in quantum yield as a function of denaturant thus defines the unfolding transition curve.

\subsubsection{Gradient Gel Electrophoresis}

Electrophoresis through polyacrylamide gels in the presence of gradient of a denaturant is a useful method for distinguishing various conformational states of proteins and for analyzing the thermodynamic and kinetic properties of transitions between conformations. The most extreme conformational transition undergone by most proteins is that between the unfolded and the native state.

Gradient gel electrophoresis measures primarily the compactness of the protein molecule as reflected in its rate of migration through polyacrylamide gel. The relative mobilities through the polyacrylamide gel thus provides qualitative estimates of compactness. The electrophoresis procedure provides a continuous two-dimensional pattern of the effect of denaturant on the shape (hydrodynamic radius) of the protein and is especially sensitive to microheterogeneity of the protein.

Protein unfolding transitions induced by denaturant are readily studied using slab gels containing a gradient of 
denaturant concentration perpendicular to the direction of electrophoresis. Protein applied across the top of such a gel migrates in the presence of continuously varying denaturant concentrations, and a profile of the unfolding transition is generated directly. The resulting pattern gives a visual illustration of the variation of protein conformation with the changing conditions.

The unfolding of the protein, caused by a denaturant, results in an increase in the hydrodynamic radius of the protein. Therefore, the unfolded and the partially-folded proteins have a reduced mobility as compared to the native state. From a single experiment, one can obtain information regarding the entire denaturant mediated transition and the overall tertiary structure of the protein.

\subsubsection{Circular Dichroism}

Circular dichroism is a most sensitive probe of the polypeptide backbone conformation of globular proteins. In the far-UV region (below $250 \mathrm{~nm}$ ) the spectral changes primarily result from the polypeptide backbone conformation, especially secondary structures, such as, $\alpha$-helix, $\beta$-turn, $\beta$ sheet and random coil. Each of these structures have CD bands between 178 and $240 \mathrm{~nm}$ (Figure 3). The CD for an $\alpha$-helix dominates this region with a reasonably intense band central about $222 \mathrm{~nm}$, and another intense band at $208 \mathrm{~nm}$. For each one of these structures there are two more CD bands between 178 and $200 \mathrm{~nm}$. In this region, the CD of the $\alpha$-helix dominates with an intense positive band at $192 \mathrm{~nm}$. Also, the $\beta$-sheet has a fairly intense band at $198 \mathrm{~nm}$ and the random coil has an intense band at $198 \mathrm{~nm}$ (Johnson, 1990). The ellipticity at $222 \mathrm{~nm}$ serves as a good measure of the $\alpha$-helix content because $\alpha$-helix is the major contributor at this wavelength. Previous studies have shown that $\alpha$-helix 


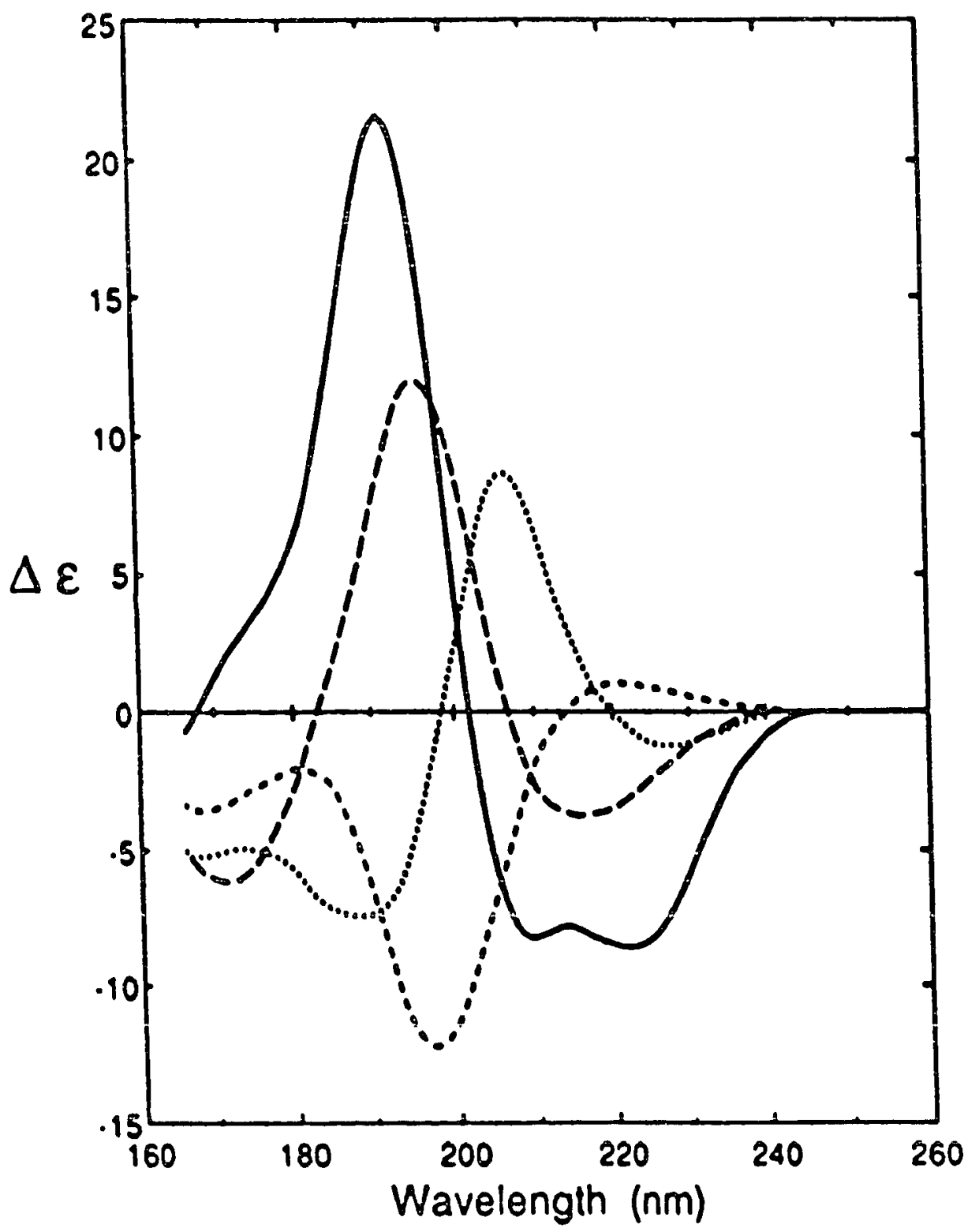

Figure 3. The CD of various secondary structures: alpha helix (-), antiparallel beta sheet $(--)$, beta turn $(\cdots \cdot \cdots)$, and random coil (--), taken from Johnson (1990). 
does not contribute to the ellipticity at $202 \mathrm{~nm}$ and all other secondary structures will contribute. Therefore, ellipticity at $202 \mathrm{~nm}$ can serve to monitor secondary structure elements other than helix. Unfortunately, the ellipticity at $202 \mathrm{~nm}$ cannot be obtained at high concentrations of all the denaturants.

\subsubsection{Hydrophobic Dye Binding}

ANS (8-anilino-1-naphthalenesulfonate) is an anionic amphiphile which, in an aqueous medium, displays a broad emission spectrum with a maximum set at $540 \mathrm{~nm}$. The ANS molecules have the ability to bind to exposed hydrophobic regions in protein and such binding results in an alteration in the optical properties of the dye, in particular, an increase in the fluorescence quantum yield (LaPorte, et. al., 1980). In the unfolded state ANS does not bind the protein, but in the native state small amount of binding is present. However, ANS binds tightly to the "hydrophobic pockets" which form when the protein is partially-folded.

ANS binds to exposed hydrophobic regions on the native protein. If these regions are "pocket-like," the microsolvent environment about the ANS molecule will be considerably more hydrophobic than if binding occured merely on the surface of the protein. In such cases, the fluorescence quantum yield will be considerably higher. ANS can also bind to the exposed hydrophobic regions on the unfolded protein molecule. Since the structure of the unfolded protein is quite expanded in this state, the average microsolvent environment about the ANS molecule will be less polar than if they were bound in hydrophobic pockets. The fluorescence quantum yields will, therefore, be lower.

Native-like intermediate states are typically slightly more expanded than the native state. The expansion generally produces more or larger hydrophobic pockets than are found in the native state. Under conditions which serve to populate such states, the average microsolvent environment about added 
ANS will be considerably more hydrophobic than under conditions which favor either native or unfolded state.

The quantum yield for ANS will, therefore, be the target under such conditions. The fluorescence quantum yield of ANS can serve to monitor the formation and decomposition of native-like intermediate states. 


\section{Chapter 2: MATERJAIS AND METHODS}

\subsection{Materials}

Bovine Pancreatic Ribonuclease A, mercaptoethanol, 1, 3dimethylurea, N-methylurea, and 8-Anilino-1-naphthalenesulfonic acid (ANS) were purchased from Sigma Chemical Co. (ST. Louis, Mo). Electrophoresis grade Tris base, dithiothreitol and sodium iodoacetate were purchased from Fisher Scientific (N.J.). Ultrapure urea and guanidinium hydrochloride were purchased from Schwartz-Mann Biotech. Ethylurea, formamide and acetamide were purchased from Aldrich Chemical Company, Inc. Dimethyl sulphoxide (HPLC grade) was purchased from J. T. Baker, Inc. (N.J.).

\subsection{Methods}

\subsubsection{Purification of Ribonuclease $A$}

Ribonuclease A (RNase A) was further purified (Biringer, 1982a) on a Sephadex SPC-25 column $(1.5 \times 25 \mathrm{~cm}, 0.15 \mathrm{M}$ phosphate buffer, $\mathrm{pH}$ 5.5). In a typical purification, a $200 \mathrm{mg}$ RNase A sample was applied to the column in 1-2 $\mathrm{mL}$ of eluting buffer. The sample was eluted with $0.15 \mathrm{M}$ phosphate, $\mathrm{pH} 6.5$ at a $0.5 \mathrm{~mL} / \mathrm{min}$ flow rate. This procedure facilitates the separation of RNase $S$ and aggregated material from RNase $A$. The monomeric protein fractions were desalted by dialyzing against water ( 3 water changes, $20 \mathrm{~L}$ carboy) in cold room. The purified material was lyophilized and stored at $20^{\circ} \mathrm{C}$.

\subsubsection{Preparation of Carboxymethylated RNase A (CM- RNase A)}

The reduction and subsequent carboxymethylation of RNase A was prepared essentially by the procedure specified by McWherter et al. (1984). 
A $20 \mathrm{mg}$ sample of RNase $A$ was dissolved in $1 \mathrm{~mL}$ of the reaction buffer $(0.5 \mathrm{M}$ Tris-HCl, $6 \mathrm{M}$ guanidine-HCl, and $2.7 \mathrm{mM}$ EDTA, $\mathrm{pH}$ 8.5) in a $10 \mathrm{mI}$ vial fitted with a septum. The solution was heated at $65^{\circ} \mathrm{C}$ for $10 \mathrm{~min}$, cooled to $50^{\circ} \mathrm{C}$ and then deoxygenated for 30 minutes by bubbling nitrogen through the solution by means of a needle inserted through the septum. Forty-nine milligrams of Dithiothreitol (DTT) was added to the solution and the reduction was allowed to proceed under a nitrogen atmosphere for four hours. The solution was cooled to room temperature and $0.14 \mathrm{~g}$ of sodium iodoacetate in $0.5 \mathrm{~mL}$ of the deoxygenated reaction buffer was added to carboxymethylate the protein. The vial was covered with aluminum foil to limit the exposure to light. The carboxy- methylation proceeded for $20 \mathrm{~min}$ and the reaction was then quenched by the addition of $2 \mathrm{mI}$ of 2mercaptoethanol. The reaction mixture was then desalted on a G-25 column equilibrated in $0.1 \mathrm{M}$ acetic acid. The protein fractions were collected, dialyzed and lyophilized.

\subsubsection{Absorbance and Fluorescence Measurements}

Absorbance measurements were performed at $286 \mathrm{~nm}$ on a Hewlett-Packard 8452A Diode Array Spectrophotometer and the fluorescence measurements were carried out with a PerkinElmer LS-3 spectrofluorometer with the excitation set at 280 $\mathrm{nm}$ and the emission set at $305 \mathrm{~nm}$. The temperature in the sample cuvettes was maintained at $17 \pm 1^{\circ} \mathrm{C}$ by an insulated and thermostated brass-block cell holder.

In a typical experiment, denaturant stock solution was prepared volumetrically in $0.05 \mathrm{M}$ Tris/formate buffer from which other intermediate dilutions were prepared so as to obtain a complete set of dilutions for the transition. A protein stock solution of $10 \mathrm{mg} / \mathrm{mL}(0.05 \mathrm{M}$ Tris/formate) was prepared in the same buffer. To each $1900 \mu \mathrm{L}$ sample of denaturant solution, $100 \mu \mathrm{L}$ of the protein stock solution was added so that final volume was $2 \mathrm{~mL}$. Matched reference samples were also prepared. The absorbance and fluorescence 
were measured after allowing the samples to reach equilibrium for one hour.

The precise protein concentration of the stock solution was determined spectrophotometrically in $0.1 \mathrm{M}$ acetate buffer at $\mathrm{pH}$ 6.0. A molar absorptivity of $9800 \mathrm{Au} / \mathrm{M}$ at $278 \mathrm{~nm}$ was used.

\subsubsection{Unfolding Monitored by circular dichroism (CD)}

CD spectra were taken on a Cary 61 spectropolarimeter under constant nitrogen flush. The pathlength of the cell (optical cuvette) used was $0.0125 \mathrm{~mm}$. All measurements were carried out at $17 \pm 1^{\circ} \mathrm{C}$. The temperature was controlled by a refrigerated water circulating water bath. The instrument was calibrated with (t)-10-camphorsulfonic acid. No skew in the molar ellipticity was observed (Johnson, 1990). The spectra were obtained from $260 \mathrm{~nm}$ to the lowest wavelength possible. At high denaturant concentrations it is not possible to obtain the spectra in the far UV due. to background absorbance of the denaturant.

The protein concentrations for $C D$ measurements were typically $5 \mathrm{mg} / \mathrm{ml}$. The protein solutions were filtered through a $0.5 \mu \mathrm{M}$ Millipore filter to remove any dust particles. The protein concentration was determined spectrophotomatically as noted above.

In a typical experiment, a protein stock solution of $50 \mathrm{mg} / \mathrm{ml}$ was prepared in the buffer $10.05 \mathrm{M}$ Tris/formate, $\mathrm{pH}$ 4.0) and this stock solution was further used to prepare two other stock solutions ( $0 \mathrm{M}$ and high molarity of the denaturant) so that final concentration of the protein in the sample is $5 \mathrm{mg} / \mathrm{ml}$. These two stock solutions were used to prepare the intermediate dilutions so as to obtain a complete set of dilutions for the whole transition. The blank samples were prepared in a similar manner.

\subsubsection{Gradient Gel Electrophoresis}

Denaturant gradient gel electrophoresis was performed to 
obtain the information regarding the protein's overall tertiary structure. The migration of protein through polyacrylamide gels depends upon the hydrodynamic radius, size and the number of charged groups. Hence, it is a measure of the relative compactness of the molecule. In the presence of the denaturant, the migration rate will depend whether the protein is folded, partially folded or unfolded.

Denaturant-gradient gels $(16 \times 13 \times 0.5 \mathrm{~cm})$ were prepared by photopolymerizing the acrylamide solutions in an in-house manufactured slab gel casting apparatus in which a gradient of denaturant had been formed by mixing two acrylamide solutions with the two limiting concentrations of denaturant. Each solution was buffered at $\mathrm{pH} 4$ with $0.05 \mathrm{M}$ Tris/formate.

In DMSO gradient gels, the first solution contained $19 \%$ acrylamide, 30\% glycerol and no DMSO, and the second solution contained 15\% acrylamide, no glycerol, and 75\% DMSO; the other components are identical. A small amount of the first solution, sufficient to fill the top $1 \mathrm{~cm}$ of the gel, is first pumped into the apparatus. The gradient is then generated by pumping from low concentration solution into the slab gel, using a two chamber mixing device, while simultaneously mixing the high concentration solution with the low concentration solution. After the gradient is formed, more of the high concentration solution is pumped in, so that the gel is finally composed of a $1 \mathrm{~cm}$ length of the low concentration solution, the gradient, and a $1 \mathrm{~cm}$ length of the high concentration solution. The gels were over-layed with isobutanol (to prevent the inhibition of polymerization of acrylamide by oxygen). DMSO gels contain acrylamide and glycerol gradients to compensate for the increased viscosity of DMSO. The solutions were deaerated under reduced pressure with an aspirator before casting the gel.

The gels contained $5 \mathrm{mg} /$ liter riboflavin and $0.12 \%(\mathrm{v} / \mathrm{v})$ $N, N, N^{\prime}, N^{\prime}$-tetramethylethylenediamine (TEMED) as polymerization catalysts. These catalysts alone will cause the 
polymerization of the gels of relatively high acrylamide concentration $(>10 \%)$ and low $\mathrm{pH}(<5)$ in about 15 minutes (Creighton, 1986). The light from two 5 watt "cool white" fluorescent tubes was used to photopolymerize the gels; consequently, polymerization can be prevented until after the gradient is formed.

After photopolymerization, the DMSO slab gels were inserted into the electrophoresis apparatus so that the DMSO gradient was perpendicular to the direction of electrophoresis. The electrophoresis buffer used was $0.05 \mathrm{M}$ Tris-formate at $\mathrm{pH} 4.0$.

The gels were pre-electrophoresed for 1 hour at $80-90 \mathrm{~V}$, $10 \mathrm{~mA}$, and constant power. On top of the gel, $75 \mu \mathrm{l}$ of RNase $A$ solution ( $2 \mathrm{mg} / \mathrm{mL}$ in $0.05 \mathrm{M}$ Tris/formate buffer, $10 \%$ glycerol) and $75 \mathrm{ml}$ of CM-RNase $\mathrm{A}$ solution $(2 \mathrm{mg} / \mathrm{mL}$ in $0.05 \mathrm{M}$ Tris/formate buffer, $10 \%$ glycerol) were loaded. A tracking dye (pyronin $G$ ) was added with the sample to monitor both application of the sample and the progress of the electrophoresis. The sample was carefully dispensed from a microsyringe along the top of the gel.

Elertrophoresis was performed at $17 \pm 1^{\circ} \mathrm{C}, 400$ watts, and a current of $40 \mathrm{~mA}$ for four hours. After completion of electrophoresis, the gels were fixed in 50 \% TCA (Trichloroacetic acid). The gels were stained overnight in a fresh solution of $0.1 \%(\mathrm{w} / \mathrm{v})$ coomassie blue $\mathrm{R} 250$ in $10 \%(\mathrm{w} / \mathrm{v})$ TCA and $10 \%$ sulfosalicylic acid. The gels were destained by diffusion against $5 \%(\mathrm{v} / \mathrm{v})$ methanol and $7.5 \%(\mathrm{v} / \mathrm{v})$ acetic acid.

Urea gradient gel electrophoresis was performed in a similar manner at $\mathrm{pH}$ 4.0. For urea gradient gels, the first solution contained 15\% acrylamide, no urea and second solution contained $14 \%$ acrylamide and $8 \mathrm{M}$ urea. The other components were same. The gels were pre-electrophoresed for one hour at 50-60v, 10mA, and constant power. Electrophoresis was performed at $17 \pm 1^{\circ} \mathrm{C}, 30$ watts constant power, and a current of $50 \mathrm{~mA}$ for 3 to 4 hours. Staining and destaining of 
gels was carried out as described above for DMSO gels. Attempts were made to pour gels with other denaturants, but due to solubility limitations, usable gels could not be produced.

\subsubsection{Hydrophobic Dye Binding Measurements}

Interaction between RNase $A$ and a hydrophobic fluorescent probe (8-anilino-1-naphthalenesulfonate) was examined in order to determine if RNase $A$ expresses hydrophobic binding sites in the presence of a denaturant. The hydrophobic dye binding measurements were carried out with a Perkin-Elmer LS-3 spectrofluorometer with the excitation set at $400 \mathrm{~nm}$ and the emission set at $470 \mathrm{~nm}$ at $17 \pm 1^{\circ} \mathrm{C}$. The temperature in the sample cuvette was maintained by an insulated and thermostated brass-block cell holder.

In a typical experiment, samples were prepared as noted above for the absorbance and fluorescence experiments with the following exceptions: 1) A protein stock solution of 1 $\mathrm{mg} / \mathrm{ml}$ was prepared in the same buffer, and 2) to each sample, $100 \mu \mathrm{L}$ of protein ( $1 \mathrm{mg} / \mathrm{mL})$ and $100 \mu \mathrm{L}$ of ANS $(20 \mathrm{mg} / \mathrm{mL})$ was added so that the final ANS to protein ratio was $20: 1$. The samples were allowed to reach equilibrium for one hour.

\subsubsection{Denaturation Curve Analysis}

The data obtained from all the experiments was analyzed with Excel ${ }^{\mathrm{TM}}$ (a spread-sheet program). Absorbance, fluorescence, Rf values (in the case of denaturant gels), and circular dichroism data was analyzed as follows:

Assumption of two-state mechanism was made where

$\mathrm{N}<---->\mathrm{U}$

only the native state, $N$, and the unfolded state, $U$, are present at significant concentrations in the transition region(Pace, 1986). Unfolding is followed by the variation of an observable parameter $Y$, where $Y_{N}$ is characteristic of the 
native state and $Y_{U}$ is characteristic of the unfolded state. Since the values for $Y_{N}$ and $Y_{U}$ are typically linearly dependent on denaturant concentration, a normalized transition can be obtained by extrapolation of the linear portions of raw data at high and low denaturant concentrations across the transition region (Figure 4).

For a two-state mechanism, $F_{N}+F_{U}=I$, and $Y=Y_{N}{ } F_{N}+$ $Y_{U}{ }^{*} F_{U}$ where $F_{N}$ and $F_{U}$ represent the fraction of the protein present in the native and unfolded states, respectively. $F_{N}$ and $F_{U}$ can be calculated by combining these equations,

$F_{N}=\left(Y_{U}-Y\right) /\left(Y_{U}-Y_{N}\right)$
$F_{U}=\left(Y-Y_{N}\right) /\left(Y_{U}-Y_{N}\right)$

Subsequent plotting of the fraction of unfolded protein versus denaturant concentration gives sigmoidal shape curve from which the $\mathrm{cm}$ (midpoint of the transition) values can be calculated.

Also, an equilibrium constant, $K_{e q}$ and a free energy of unfolding, $\Delta G_{D}$, can be calculated as follows:

Keq $=F_{U} / F_{N} \quad$ and

$\Delta G_{D}=-R T$ In $(\mathrm{Keq})$

Therefore

$\Delta G_{D}=-R T$ In $\left(F_{U} /\left(1-F_{U}\right)\right)$

Theoretical and experimental evidence suggests that for a simple two state transition the $\Delta G^{a O}$ values are linearly dependent on denaturant concentration (Green \& Pace, 1974; Ahmad \& Bigelow, 1982; Schellman, 1978). The linear relation 


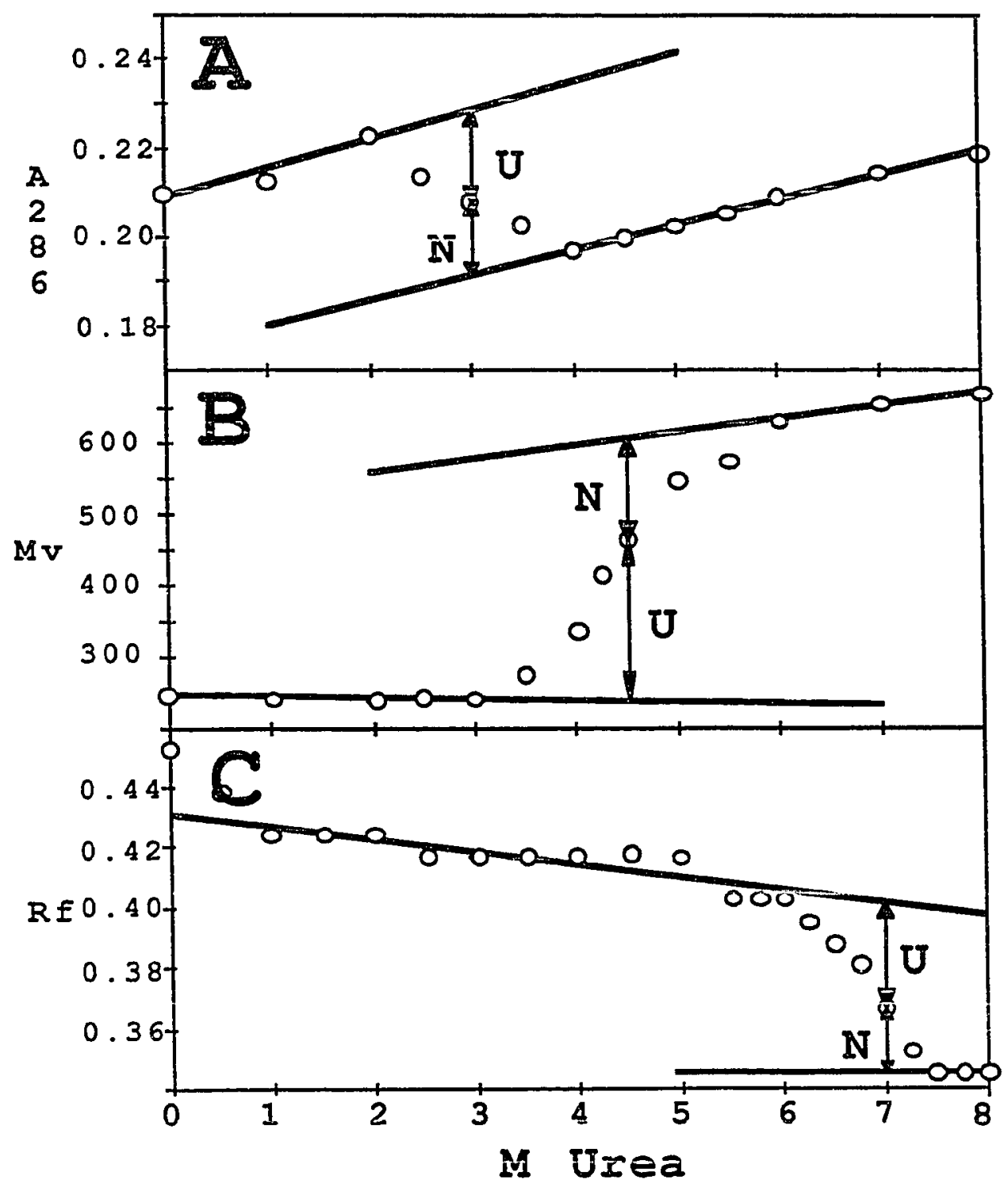

Figure 4. Extrapolation of baselines across the urea-induced unfolding transition $\left(\mathrm{pH} 4,17 \pm 1^{\circ} \mathrm{C}\right.$ ). $\mathrm{N}$ and $\mathrm{U}$ represent the relative amounts of native and unfolded protein, respectively. A) Absorbance at $286 \mathrm{~nm}$, B) fluorescence $(280 / 305 \mathrm{~nm})$,

C) Urea-gradient gel. 
is described by equation $\sigma$ where $\Delta G^{\text {aO }}$ is the free energy change for the transition in the absence of denaturant.

$\Delta G_{D}=\left(\Delta G_{D} / M\right.$ denaturant $) \quad M$ denaturant $+\Delta G^{a O}$

The $\Delta G^{\text {ao }}$ values were obtained from a linear regression analysis of $\Delta G_{D}$ vs denaturant data. Standard deviation and correlation coefficient were calculated for each of the analysis.

\subsubsection{The Analysis of ANS Binding Curve}

ANS binding curves are typically Gaussian in shape. The simplest model which describe this situation is as follows:

$$
\mathrm{A}<--\mathrm{K}_{1}--->\mathrm{B}<---\mathrm{K}_{2}---->\mathrm{C}
$$

where $K_{1}$ and $K_{2}$ are the equilibrium constants for the respective processes.

The ANS data was curvefitted with NFIT (nM (nonlinear least squares curve fitting program) by using the following equation which is based on the simple model:

$$
\begin{aligned}
& ((Z 0 / \operatorname{EXP}(-1 / R T *(X * S 1+D G 1))))+Z 1) /((\operatorname{EXP}(-1 / R T *(X * S 1+D G 1))) \\
& +1+(\operatorname{EXP}(-1 / R T *(X * S 2+D G 2)))))+(Z 2 /((\operatorname{EXP}(-1 / R T *(X * S 1+D G 1+X \\
& * S 2+D G 2)))+1+(\operatorname{EXP}(-1 / R T *(X * S 2+D G 2)))))
\end{aligned}
$$

where

- DG1 and $S_{1}$ are $D G^{a O}$ and $D G_{D} / M$ denaturant for the equilibrium defined by $\mathrm{K}_{1}$.

- DG2 and $S 2$ are $D G^{a o}$ and $D G_{D} / M$ denaturant for the equilibrium defined by $\mathrm{K}_{2}$.

-20, 21 , and $\mathrm{Z2}$ are the relative molar fluorescence value of $A, B$, and $C$.

- $\mathrm{X}=\mathrm{M}$ of denaturant. 


\section{Chapter 3: RESUITS AND DISCUSSION}

Denaturant-induced protein folding can be followed as a function of denaturant concentration by use of any observable parameter which gives a detectable difference between the native and the unfolded states of the protein. The unfolding of RNase A was monitored by absorbance, fluorescence, circular dichroism, denaturant-gradient gel electrophoresis, and hydrophobic dye binding at $\mathrm{pH}$ 4. Each one of these techniques monitors different properties of the protein during the unfolding processes. By comparing the data from differeni techniques, one can determine if there are any intermediate structures present in the unfolding process and glean information about their structure and relative stabilities.

\subsection{Rbsorbance and Fluorescence}

The denaturant-induced unfolding transitions were determined for RNase $A$ at $p H 4.0$ in each denaturant by monitoring the change in absorbance at $286 \mathrm{~nm}$ and the change in fluorescence with the excitation set at $280 \mathrm{~nm}$ and the emission set at $305 \mathrm{~nm}$.

As the protein is subjected to increasing concentrations of denaturant, an increase in the fluorescence and decrease in absorbance is observed ( $E$ igure 5 ). These changes are only observed when the protein unfolds. A small linear increase in absorbance is observed before and after the transition, for all the denaturants. In case of fluorescence, a few denaturants, such as formamide and ethylurea, showed quenching of the fluorescence before and after the transition. These changes can be attributed to the solvent perturbation effect of denaturant on the exposed tyrosines in the native and the unfolded conformations, respectively. 

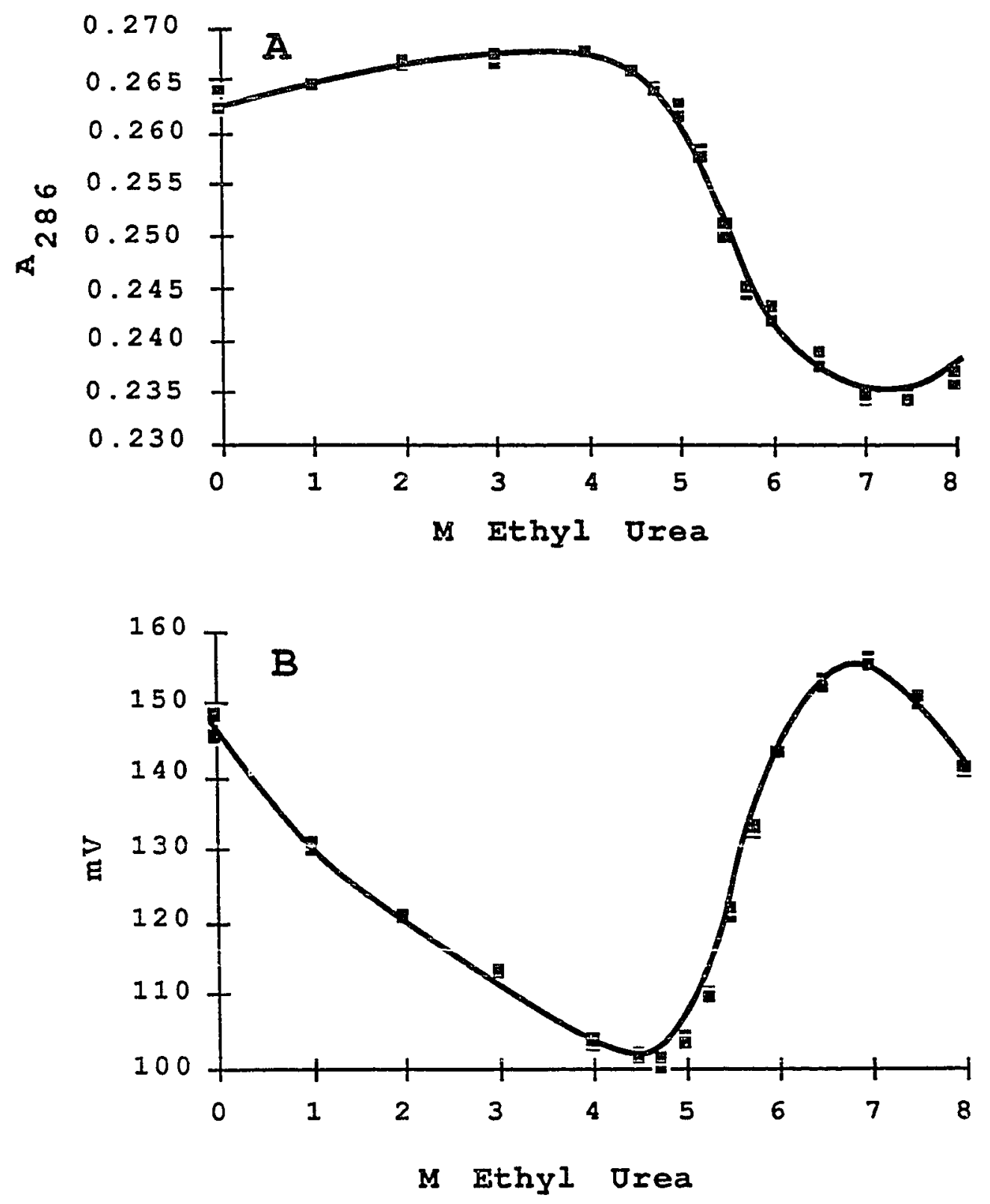

Figure 5. A) Absorbance (286 nm) and B) Fluorescence $(280 / 305 \mathrm{~nm})$ of RNase A as a function of M Ethyl Urea concentration at $\mathrm{pH} 4.0$ and $17 \pm 1^{\circ} \mathrm{C}$. 
Subsequent plotting of the fraction of unfolded protein versus concentration gave sigmoidal curves from which the $\mathrm{Cm}$ values were calculated (Figure 6). A plot of the change in free energy of transition as a function of denaturant concentration (Figure 7) allows the calculation of the $\Delta G^{a}$ of the transition.

\subsubsection{Effect of Denaturation on Absorbance and FIuorescence}

In all the denaturants, it was observed that transition curves obtained by absorbance and fluorescence were coincident with each other. Therefore, only the results obtained from fluorescence will be discussed in more detail. Figure 8 shows the fraction unfolded curves for all the denaturants. Evidently, the transition curves shift to a higher denaturant concentration with respect to the number of hydrogen bond donors on the denaturant molecule. In addition, the transitions become less cooperative with urea and urea derivatives such as N-methylurea, ethylurea and 1, 3 dimethylurea. This indicates that intermediate structures can be populated with weaker denaturants. However, transitions for formamide and acetamide are as cooperative as urea; reasons for this are unknown at this time.

The values for the midpoints of the transitions, $\mathrm{Cm}$, and the corresponding change in apparent free energy, $\Delta G^{a 0}$, are given in Table-1. Comparison of the $\mathrm{cm}$ values for fluorescence confirms that loss of one hydrogen bond donor decreases the denaturant strength to a small extent, but loss of one hydrogen bond acceptor decreases the strength of the denaturant a significant amount as is observed in the case of acetamide and formamide.

The change in $\Delta \mathrm{G}^{\mathrm{a} O}$ is variable with respect to the strength of the denaturant. For urea and its derivatives, the $\Delta G^{a 0}$ appears to decrease with increasing $\mathrm{Cm}$. The relationship between $\mathrm{Cm}$ and $\Delta \mathrm{G}^{\mathrm{a} O}$ is dependent on the cooperativity of the 
Fig $6 \& 7$ 


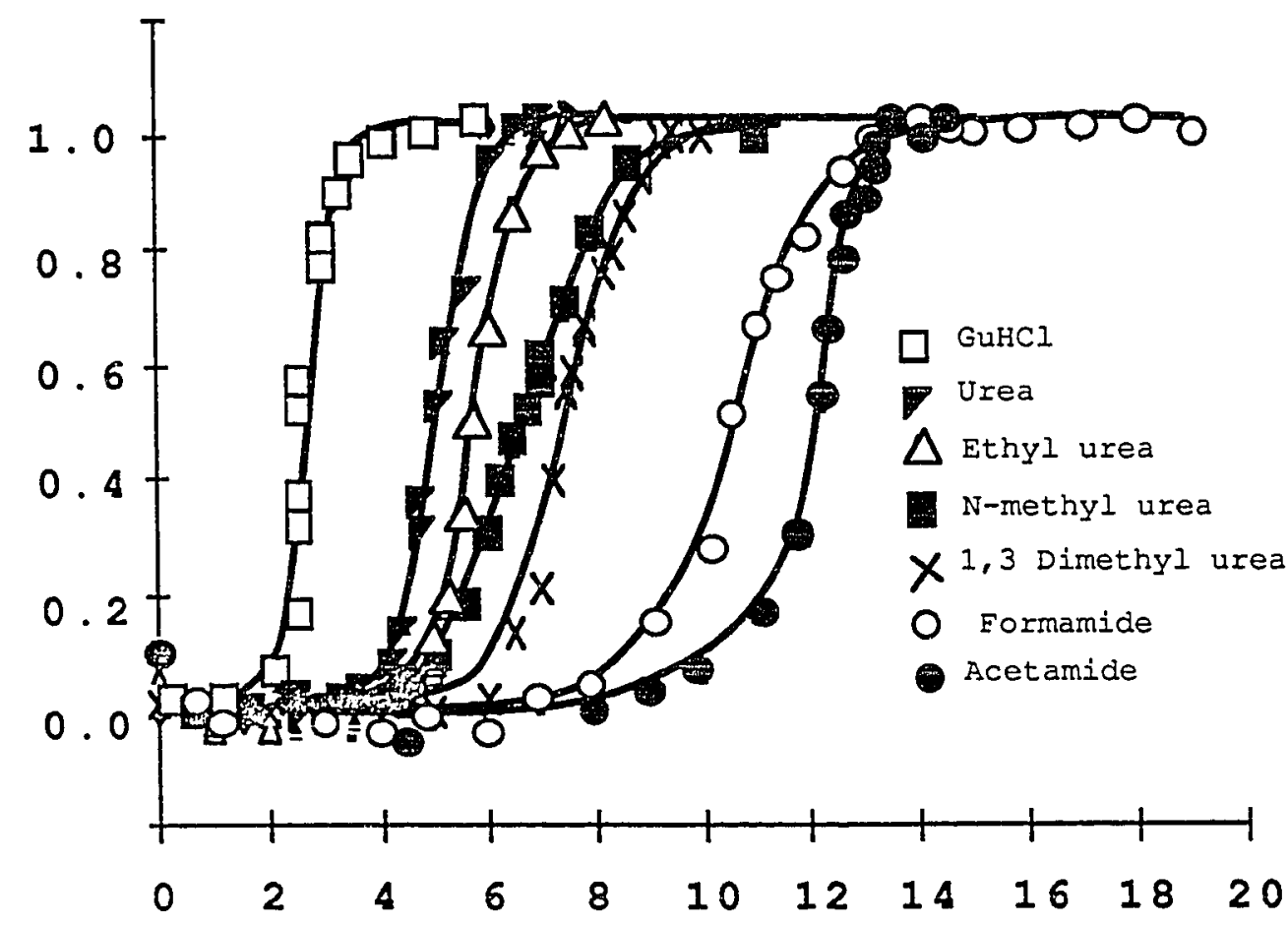

M Denaturant

Figure 8. Eraction of unfolded RNase A from the fluorescence data as a function of denaturant concentration for different denaturant at $\mathrm{pH} 4.0$ (Tris/formate) and $17 \pm 1^{\circ} \mathrm{C}$. 
transition as shown in equation 6 .

TABIE 1: Transition midpoints and $\Delta \mathrm{G}^{\mathrm{a} O}$ values for the denaturant induced unfolding of RNase $A$

\begin{tabular}{llll}
\hline \multicolumn{1}{c}{ Denaturant } & \multicolumn{2}{c}{ Flu } & CD \\
\hline & Cm & $\Delta \mathrm{G}^{\mathrm{aO}}$ & $\mathrm{Cm}$ \\
\hline GuHCl & 2.78 & 7.38 & 2.70 \\
urea & 5.05 & 8.20 & 6.55 \\
ethylurea & 5.76 & 8.72 & 8.12 \\
N-methylurea & 6.87 & 5.33 & 8.73 \\
1,3-dimethylurea & 7.32 & 5.63 & --- \\
formamide & 10.59 & 9.49 & 11.94 \\
acetamide & 12.39 & 27.27 & 13.52 \\
\end{tabular}

Transition midpoints $(\mathrm{Cm})$ and apparent free energy $\left(\Delta \mathrm{G}^{\mathrm{a} 0}\right)$ for RNase $A$ in various denaturants at $\mathrm{pH}$ 4.0. Values are given for fluorescence measurements at $305 \mathrm{~nm}$ with 280 excitation and molar ellipticity at 222 nm.

$\Delta G_{D}=\left(\Delta G_{D} / M\right.$ denaturant $) * M$ denaturant $+\Delta G^{a 0}$

or $\Delta \mathrm{G}_{\mathrm{D}}=$ slope $\star \mathrm{M}$ denaturant $+\Delta \mathrm{G}^{\mathrm{ao}}$

The slope, $\left(\Delta G_{D} / M\right.$ denaturant $)$ is a measure of cooperativity. High slope indicates high cooperativity and low slope indicates low cooperativity. Since the $\mathrm{Cm}$ is the molarity of denaturant when $\mathrm{keq}=1$, then $\Delta \mathrm{G}_{\mathrm{D}}=0$ at this point, thus,

$0=$ slope* $\mathrm{Cm}+\Delta \mathrm{G}^{\mathrm{aO}}$

$\Delta G^{a O}=-$ slope $* \mathrm{Cm}$

Eor transitions with identical cooperativity (identical slope) $\Delta G^{\text {ao }}$ should increase linearly with respect to $\mathrm{Cm}$. Nmethylurea, 1,3-dimethylurea and acetamide do not follow this trend but others apparently do (see Table 1). 
A change in cooperativity can influence the relationship between $\mathrm{Cm}$ and $\Delta \mathrm{G}^{\mathrm{a}}$. In order to take cooperativity into account, a plot of -Slope $v s \mathrm{Cm}$ is shown in Figure. 9. The plot clearly shows that the slope, hence, the cooperativity decreases with increasing $\mathrm{Cm}$ for GuHCl, urea, and urea derivatives and thus accounts for the apparent discrepancy. In addition, since a decrease in cooperativity indicates the population of intermediate states, urea and its derivatives facilitate the stabilization of intermediate structures. Furthermore, stabilization is inversely dependent on denaturant strength.

\subsection{Circular Dichroism}

The change in secondary structure (particularly alpha helix) was determined for each denaturant by measuring the ellipticity at $222 \mathrm{~nm}$. At this wavelength, the major contributor is alpha-helix and thus ellipticity at $222 \mathrm{~nm}$ serves as a good measure of the alpha-helix content. Since this is a negative band, an increase in the molar ellipticity is observed as the protein unfolds.

\subsubsection{Effect of Denaturant on Circular Dichroism}

Figure 10 shows the transitions obtained from all the denaturants at $\mathrm{pH}$ 4. With some of the denaturants (ethylurea, N-methylurea, DMSO, acetamide, I,3-dimethylurea) a complete transition was not observed due to the solubility limitations. Previous studies have shown that GuHCl completely unfolds the protein. Therefore, to calculate the $\mathrm{Cm}$ values for the above mentioned denaturants, the assumption was made that the completely unfolded protein has same ellipticity as protein unfolded with GuHCl. The relative shapes of the curves in Figure 10 shows that the transitions become less cooperative with decreasing number of hydrogen bond donors and hydrogen bond acceptors on the denaturant molecules. $\Delta G^{\text {ao }}$ values could not be calculated for all the 


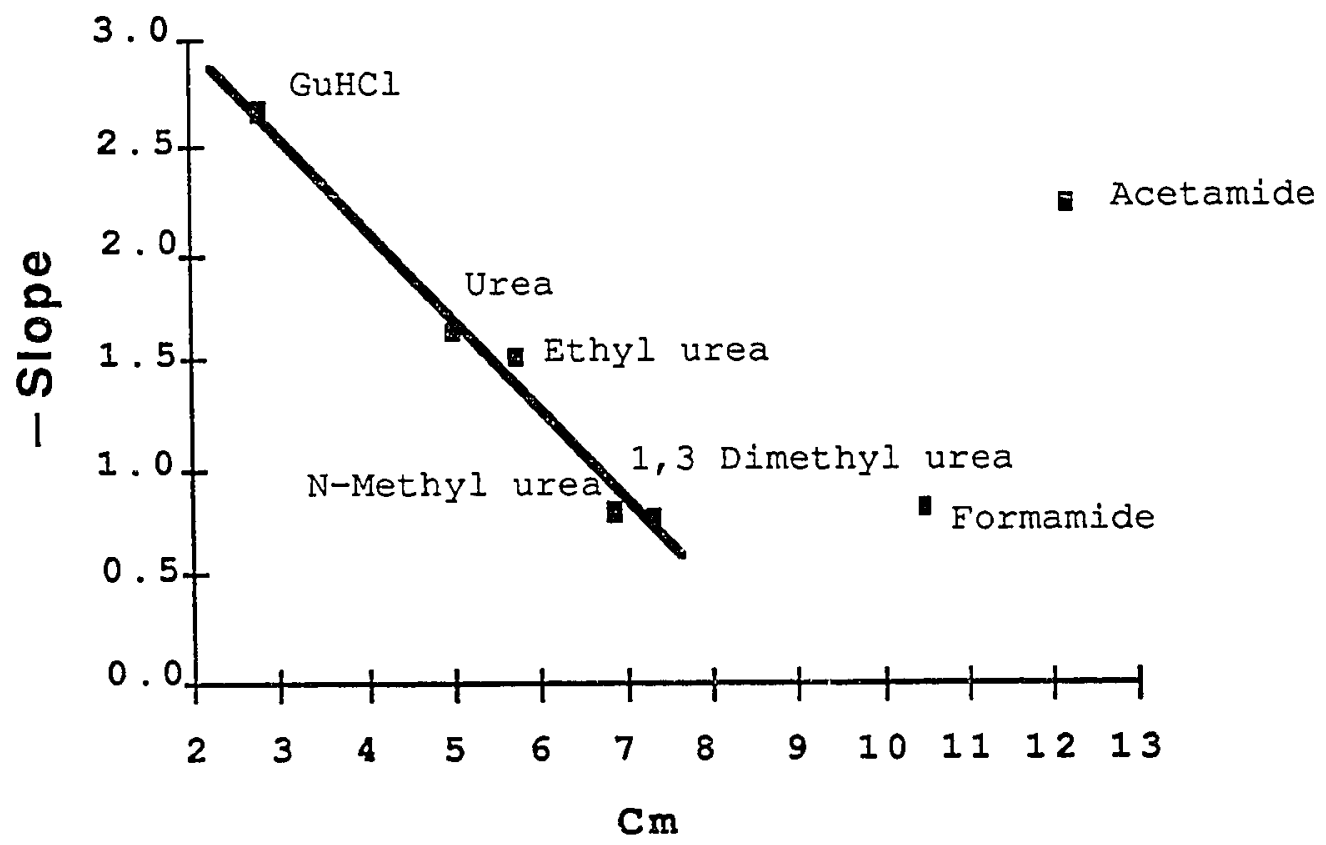

Figure 9. Slope as a function of $\mathrm{Cm}$ (midpoint of transition) from fluorescence experiments. 


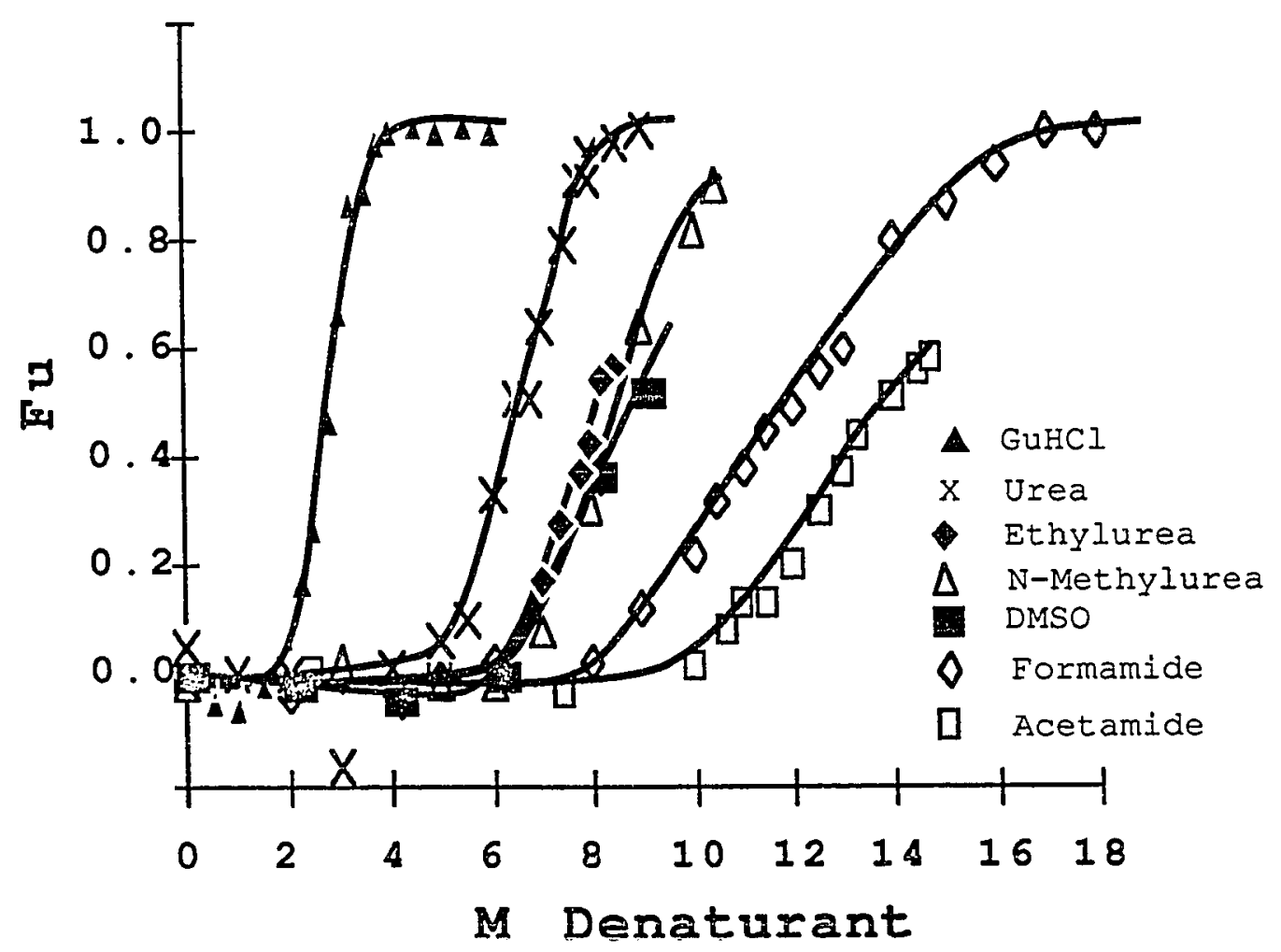

Figure 10. Eraction unfolded of RNase A observed by Circular dichroism ( $222 \mathrm{~nm})$ as a function of denaturant concentration at $\mathrm{pH} 4.0$ and $17 \pm 1^{\circ} \mathrm{C}$. 
denaturants due to the fact that the complete transitions were not observed for all the denaturants.

The $\mathrm{Cm}$ values for the circular dichroism experiments increase in the same manner as observed with absorbance and fluorescence, but occur at much higher concentration of the denaturant (Table-1). This indicates that the $\alpha$ helix structure is disrupted much later than the other structures. The only exception is the $\mathrm{Cm}$ values for GuHCl. The $\mathrm{Cm}$ values for GuHCl are same for all three techniques (absorbance, fluorescence, and $C D$ ) and thus suggests a cooperative disruption of the entire structure.

In order to compare the effect of the denaturants or: fluorescence and $C D$ transitions, the $\mathrm{Cm}$ values for the fluorescence data were plotted versus the $\mathrm{Cm}$ values for the $C D$ data (Figure 11). The plot shows a relatively linear relationship between $\mathrm{Cm}$ values obtained from both data sets. The slope of the line is essentially 1.0 which indicates that the denaturants have similar effect on both fluorescence and $C D$ transitions and hence tertiary and secondary structures, respectively.

\subsection{Hydrophobic Dye Binding}

The hydrophobic dye ANS was used in this study. Hydrophobic dyes are known to bind to hydrophobic pockets on protein surface. If such dyes are fluorescent, the quantum yield increase with binding, as the polarity of the microsolvent environment decreases. The net result is an increase in the dye's quantum yield. At low concentrations of the denaturant, proteins undergo small conformational changes and, in doing so, forms more or larger hydrophobic pockets where dye molecules can bind. At high denaturant concentration, the protein can completely unfold and, in doing so, these hydrophobic pockets are dismantled. Although the dye may still bind to exposed regions, the average microsolvent environment will be more polar than the 


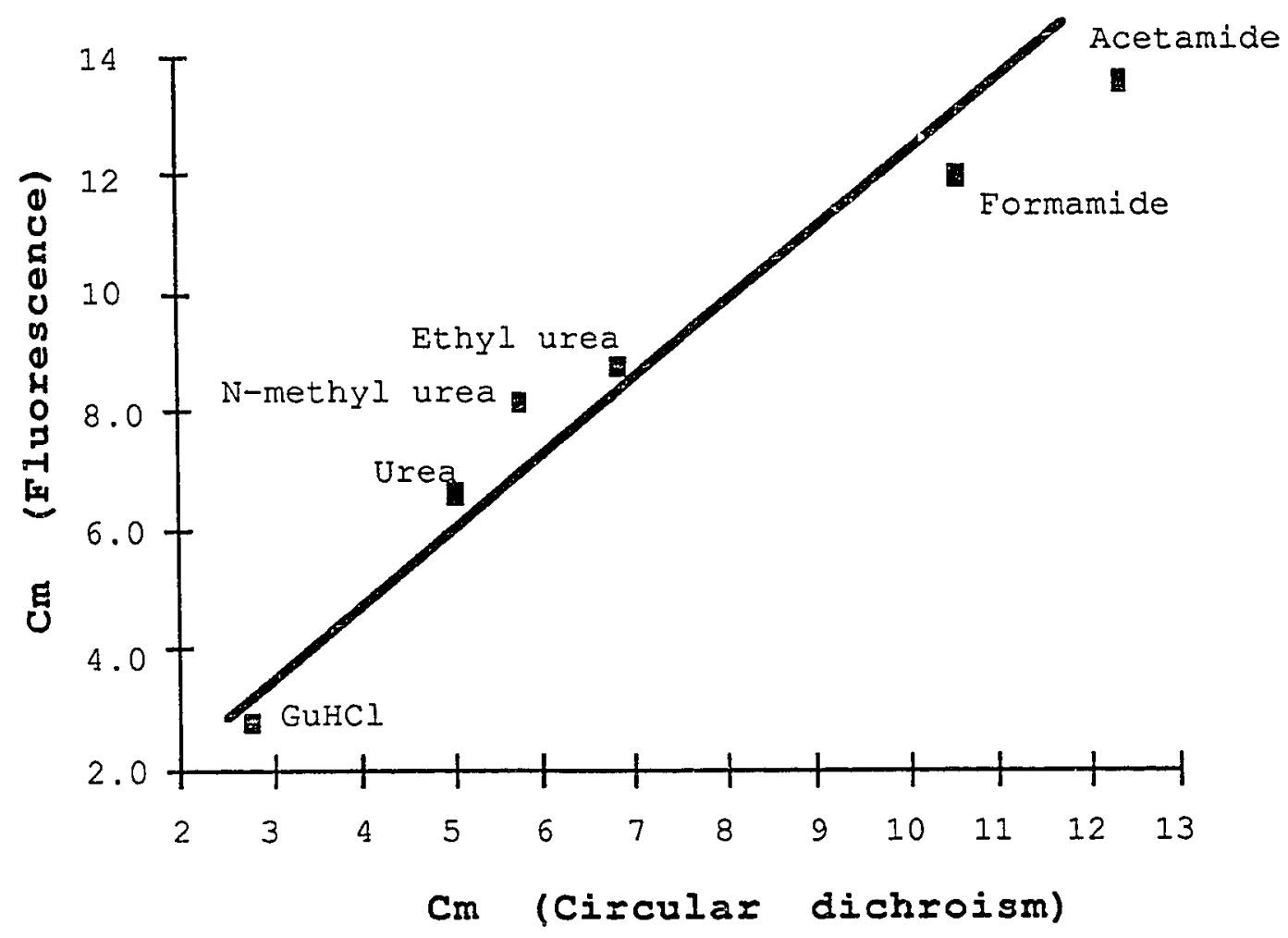

Figure 11. Cm values from fluorescence $(280 / 305 \mathrm{~nm})$ as a function of $\mathrm{Cm}$ values from Circular Dichroism (222 nm). 
partially unfolded structure.

\subsubsection{Effect of Denaturants on Hydrophobic Dye Binding}

Figure 12 shows the dual transitions observed from all the denaturants except GuHCI at $\mathrm{pH} 4$ and Table 2 lists the corres-ponding $\mathrm{Cm}$ values. Since the guanidinium ion forms a precipitate with ANS, the ANS transitions could not be obtained. Since both transitions occur at low denaturant concentration, their structures are very similar to the native protein.

TABLE 2: Transition midpoints with ANS for the denaturant induced unfolding of RNase $A$

\begin{tabular}{lcc}
\hline Denaturant & ANS 1 & ANS 2 \\
\hline urea & 2.1 & 3.8 \\
ethylurea & 1.3 & 2.8 \\
N-methylurea & 2.3 & 3.8 \\
1,3-dimethylurea & 2.3 & 3.9 \\
formamide & -- & 8.2 \\
acetamide & 5.5 & 8.2 \\
dimethyl sulfoxide & 3.3 & 5.3 \\
\hline ANS 1 is the midpoint of the first transtion and ANs 2 is the \\
midpoint of the second transition. values are given for the \\
change in the relative fluorescence at 470 nm with 400 nm \\
excitation.
\end{tabular}

The overlay plot shows that the corresponding transitions for urea, 1,3-dimethylurea, and N-methylurea are similar and others are different. Since these three denaturants differ markedly in their ability to act as hydrogen bond donors, this result suggests that their effect on protein structure at low concentrations might not involve competition for hydrogen bonds within the protein. One possibility is that these denaturants might act by displacing the surface water molecules which normally serve to stabilize the protein's structure. On the other hand, these three 


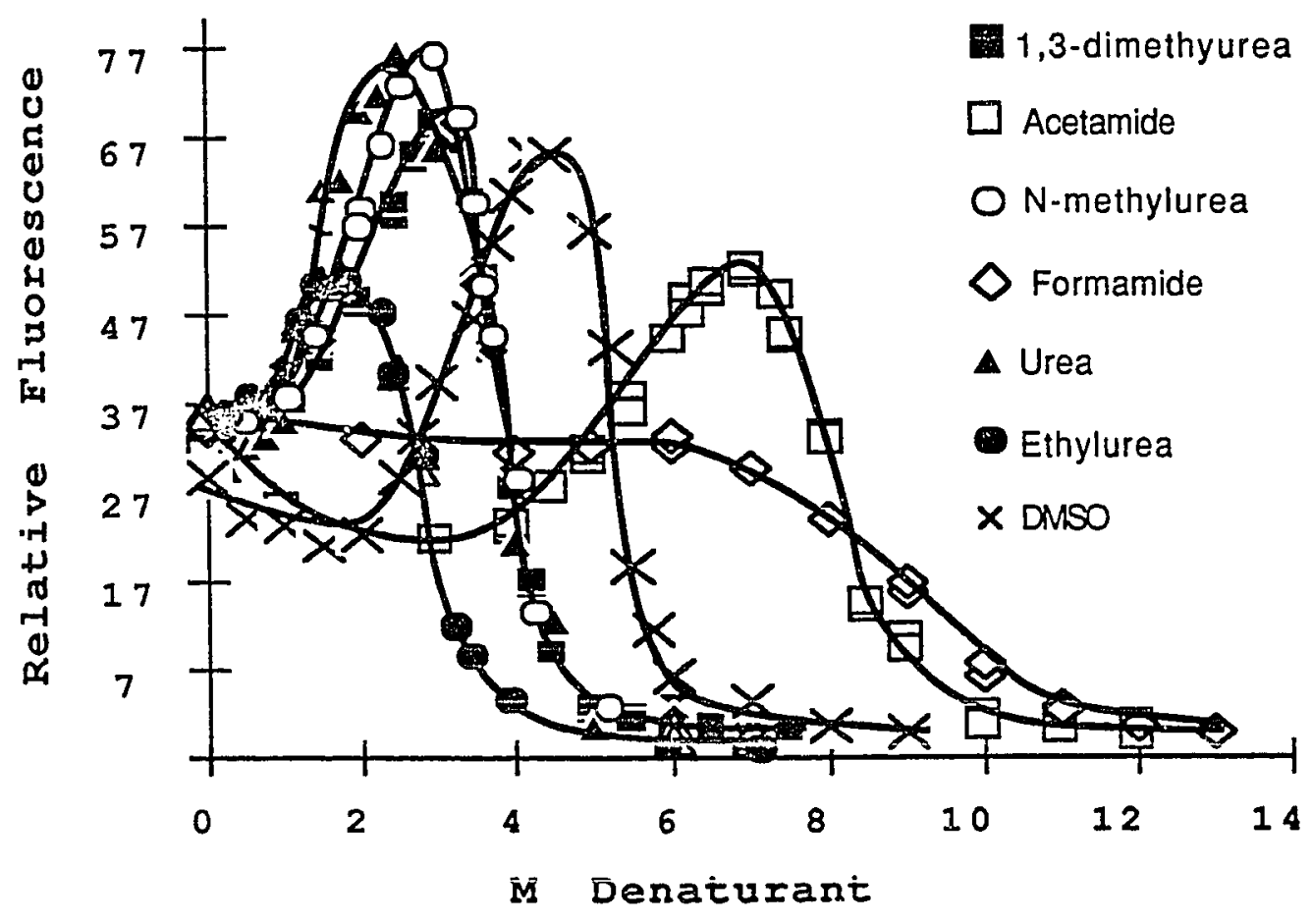

Figure 12. Fluorescence (400/470 nm) of ANS binding monitored as a function of denaturant concentration at $\mathrm{pH} 4$ and $17 \pm 1^{\circ} \mathrm{C}$. 
denaturants also differ in their hydrophobic character. For these denaturants, hydrophobicity increases as the ability to hydrogen bond decreases. Since all three denaturants have the same effect on the stability of the protein at low concentrations, it seems reasonable that both hydrogen bonding and solvation of hydrophobic groups on the protein play a role in this transition and that each is of the equal importance. The fact that hydrogen bonding ability appears to be the dominant factor in transitions observed with other techniques suggests that different hydrogen bonds are involved. One possibility is that hydrogen bonding to the surface of the protein dominates at low denaturant concentrations whereas hydrogen bonding to internal portions of the protein dominates at high denaturant concentrations. In addition, the surface interactions may result in the displacement of surface water molecules.

The results obtained for ethylurea also lends support for the importance of hydrophobic intexactions in the ANS transition. The midpoints of the transitions for ethylurea are on entire unit lower than those observed for methylurea.

The ANS transitions observed for acetamide and formamide are much higher than all others examined. Since these denaturants have the same number of hydrogens available for hydrogen bonding as 1,3-dimethylurea, but one less nitrogen for hydrogen bond accepting, it appears that the hydrogen bond acceptor ability is more important than hydrogen bond donation in the ANS transitions. The absence of the ANS 1 transition in formamide-induced unfolding requires further investigation.

DMSO is an aprotic denaturant and thus cannot act as a protein donor. The ANS transtions for this denaturant are between those obtained for one-nitrogen and two-nitrogen denaturants. Since DMSO is a stronger base than either formamide or acetamide, it is a stronger hydrogen bond acceptor. This may account for the greater denaturant strength. On the other hand, the methyl groups may aid in 
solvating hydrophobic groups on the protein.

\subsection{Denaturant-Gradient Gel Electrophoresis}

The denaturant-gradient gel electrophoresis was used to further study the effect of denaturant on the unfolding of RNase A. Usable gels could not be produced with most of the denaturants, but we were able to polymerize gels in the presence of DMSO and urea. Representative DMSO and urea gradient gels for RNase $\mathrm{A}$ at $\mathrm{pH} 4$ are shown in Figure 13 and 14, respectively.

\subsubsection{Effect of Denaturants on the Gradient-Gel Electrophoresis}

The DMSO-gradient gel pattern shows a bimodal transition (Figure 15). DMSO produced little or no change in the electrophoretic mobility of RNase $A$ up to a concentration of about $2 \mathrm{M}$, where a relatively sharp change in mobility was observed. The transiton levels off around $6 \mathrm{M}$ and then continues to $9 \mathrm{M}$. At concentrations of DMSO above this transition, little or no change in mobility occurred, which is consistent with the protein being fully unfolded. The bimodal nature of the transition indicates the presence of a stable intermediate around $6 \mathrm{M}$ DMSO which further unfolds to the unfolded state. In comparison, urea-gradient gels gave a two-state transition (Figure 16).

Figure 17 shows the results obtained from gradient gel electrophoresis and CD data for both urea and DMSO. Gel data is a monitor of tertiary structure and circular dichroism data is amonitor of secondary structure. The urea data shows that $C D$ and gel transitions are almost coincident with each other which indicates that secondary structure and tertiary structure are lost essentially simultaneously. On the other hand, DMSO shows markedly different results. The first transition shown in the DMSO gels occurs at considerably lower DMSO concentrations than that observed for the CD transition and the second is relatively coincident. Hence, it 


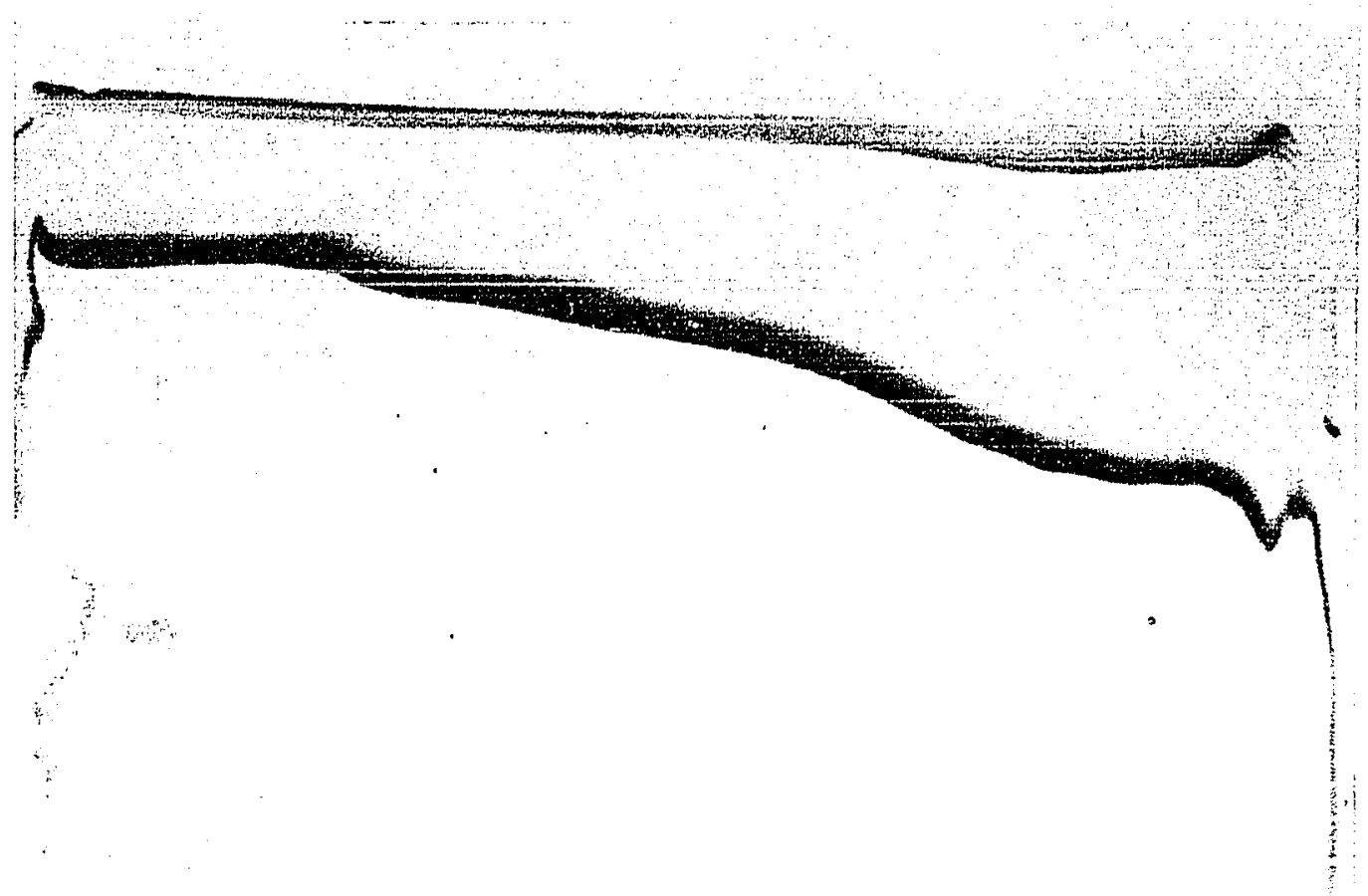

Figure 13. DMSO-gradient gel electrophoresis of RNase A at pH 4 and $17 \pm 1{ }^{\circ} \mathrm{C}$. The gradient from left to right is OM DMSO to $10.6 \mathrm{M}$ DMSO. The top most band is carboxymethylated RNase and the lower band is RNase $A$. 


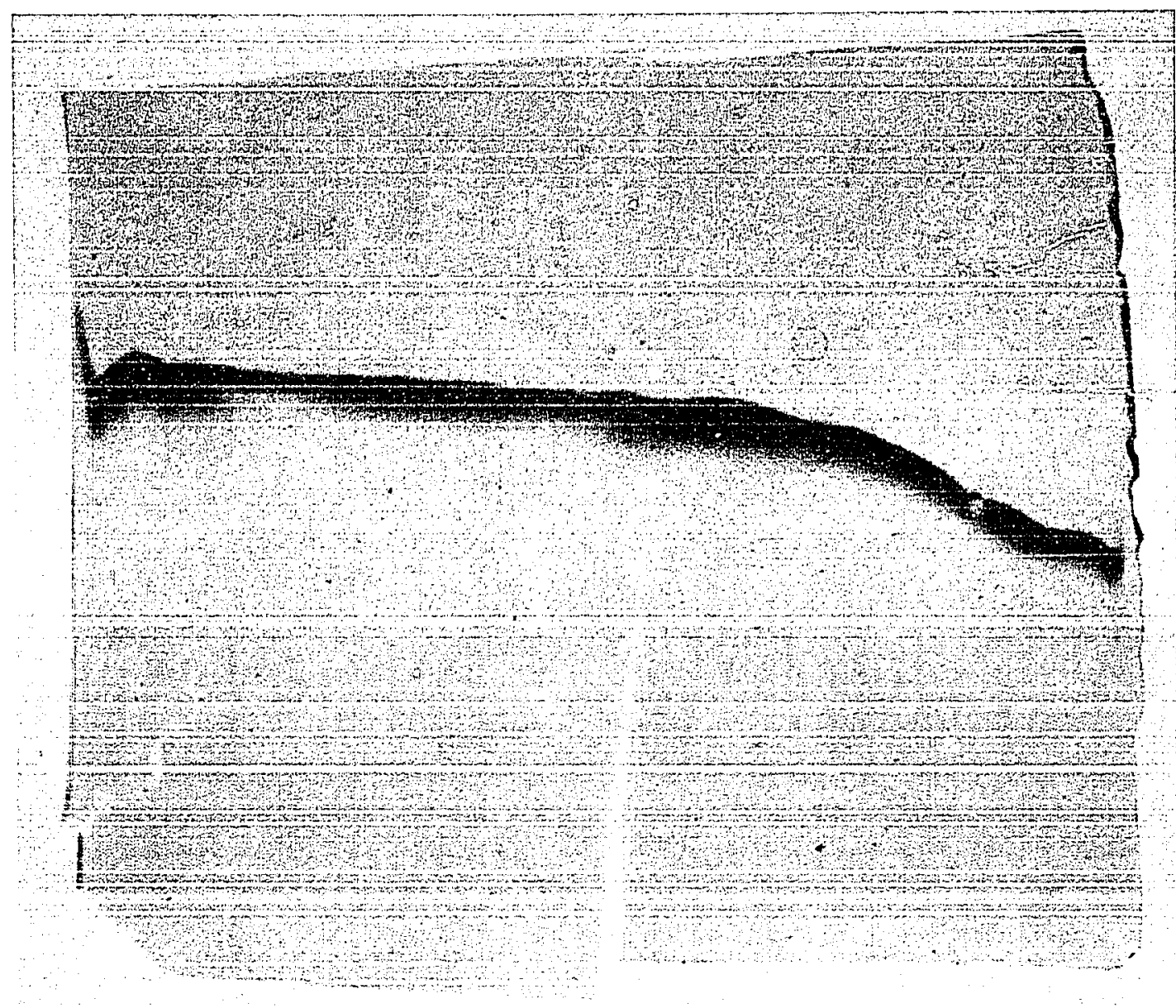

Figure 14. Urea-gradient gel electrophoresis of RNase A at pH 4 and $17 \pm 1{ }^{\circ} \mathrm{C}$. The gradient from left to right is OM Urea to $8.0 \mathrm{M}$ Urea. The top most band is carboxymethylated RNase and the lower band is RNase $A$. 


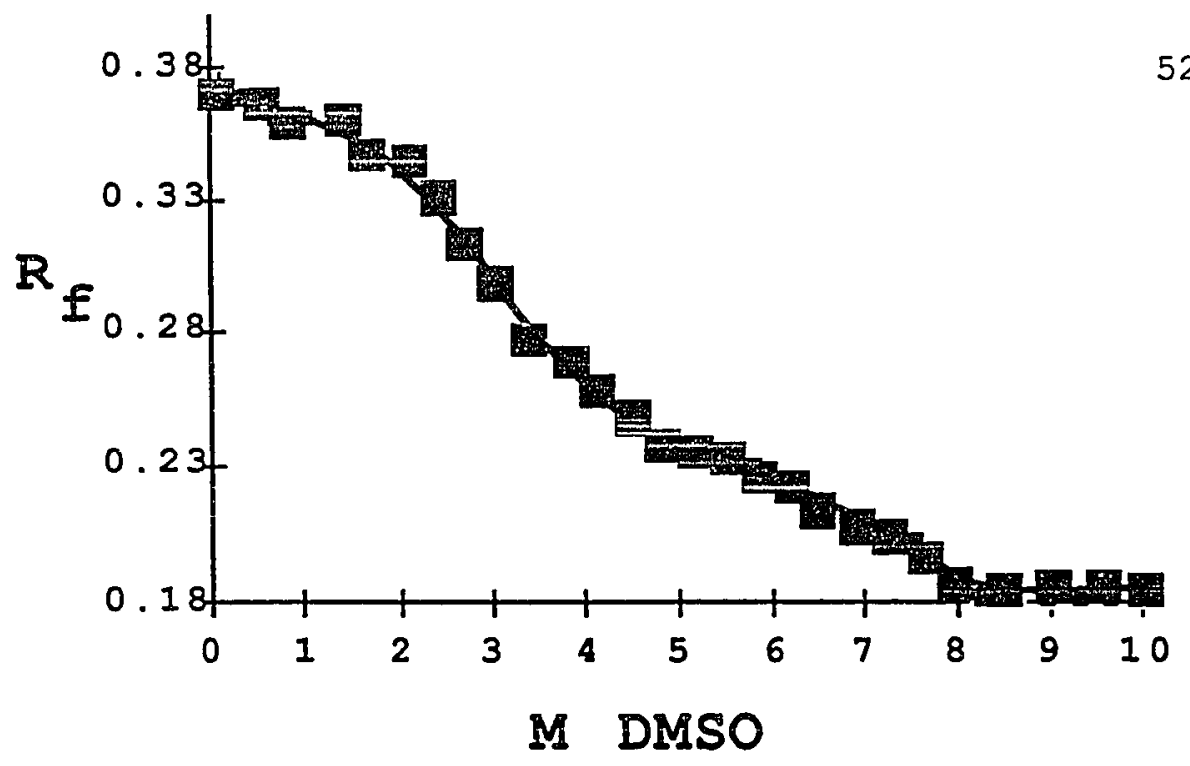

Figure 15. Relative mobility of RNase $A$ as a function of DMSO concentration at $\mathrm{pH} 4.0$ and $17 \pm 1^{\circ} \mathrm{C}$.

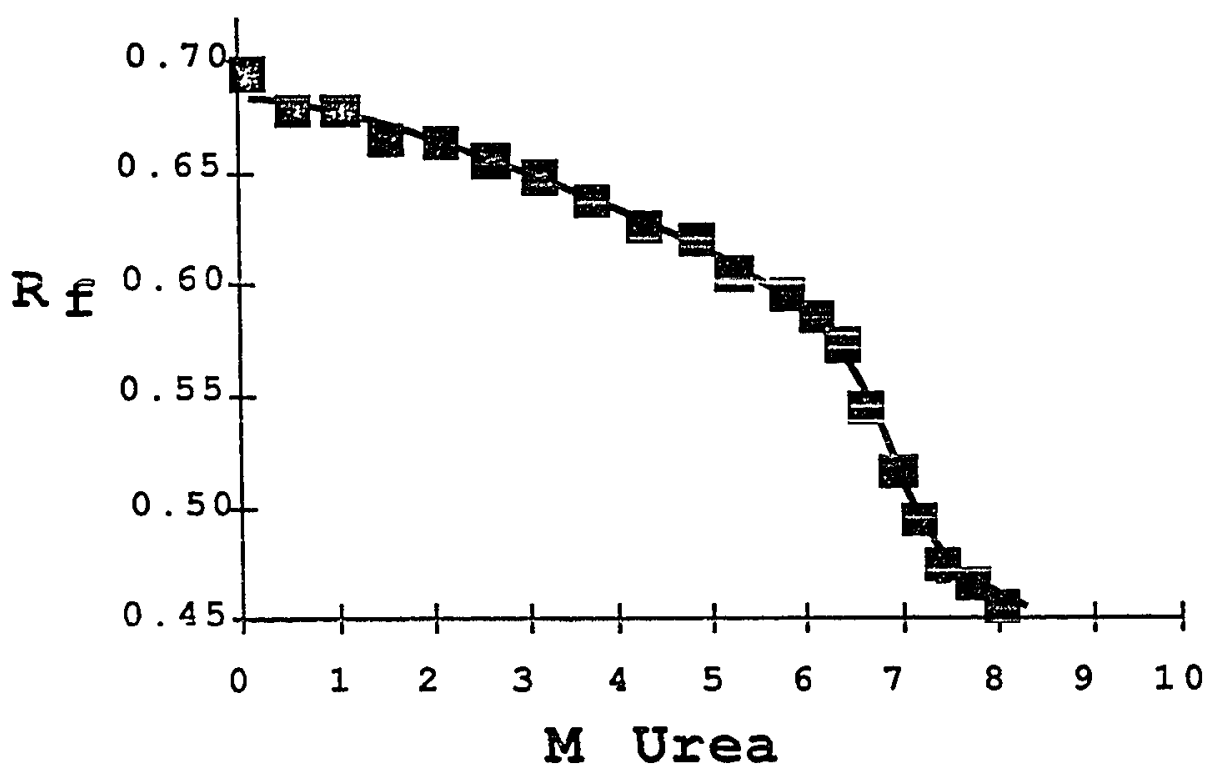

Figure 16. Relative mobility of RNase $A$ as a function of urea concentration at $\mathrm{pH} 4.0$ and $17 \pm 1{ }^{\circ} \mathrm{C}$. 


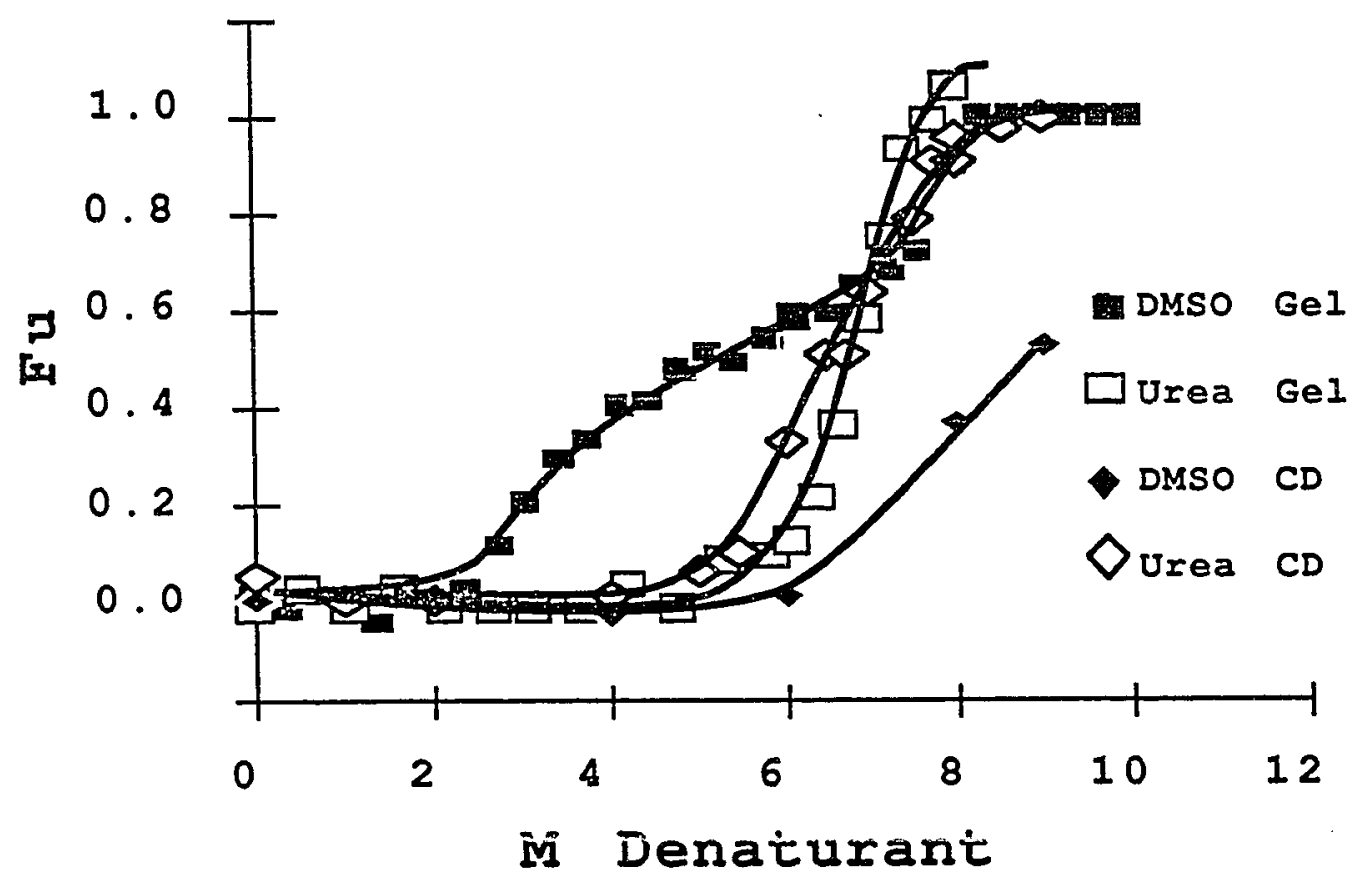

Figure 17. Fraction unfolded of RNase A vs M denaturant at $\mathrm{pH} 4.0$ and $17 \pm 1^{\circ} \mathrm{C}$. 
indicates that a significant amount of the tertiary structure of the protein is disrupted without effecting the secondary structure. Intermediate structures such as these are characteristics of molten globule states. In addition, this dramatic difference indicates that the unfolding pathway differs for the respective denaturants. The fact that DMSO disrupts the tertiary structure of the protein prior to the secondary structure strongly suggests that it acts as a hydrophobic denaturant.

\subsection{Comparison of the Data Obtained From Different Spectroscopic Techniques}

The non-coincidense of data obtained from different techniques indicates the presence of intermediate structures at denaturant concentrations between the respective transitions. With most of the denaturants, it was observed that absorbance and fluorescence transitions were coincident with each other, but these transitions were non-coincident with circular dichroism and ANS transitions. GuHCl was the only exception.

For all but DMSO and GuHCl the relation between them is the same; therefore, the data for ethylurea will be used as a model to explain the relationship between the data obtained from different spectroscopic probes. Figure 18 shows the comparison of ethylurea data obtained from absorbance, circular dichroism, fluorescence, and hydrophobic dye binding. The results clearly indicate that the P.NS binding transitions occur much earlier than the transitions observed with other techniques. These results indicate that the protein is undergoing minor structural changes in the hydrophobic regions before the secondary or tertiary structure is disrupted. The ANS transitions are indicative of the formation of intermediate structures. The ANS 1 transition indicates the formation of intermediate, $I_{1}$, and the ANS 2 transition indicates its decomposition. Since the protein is not yet fully unfolded, it decomposes into a 


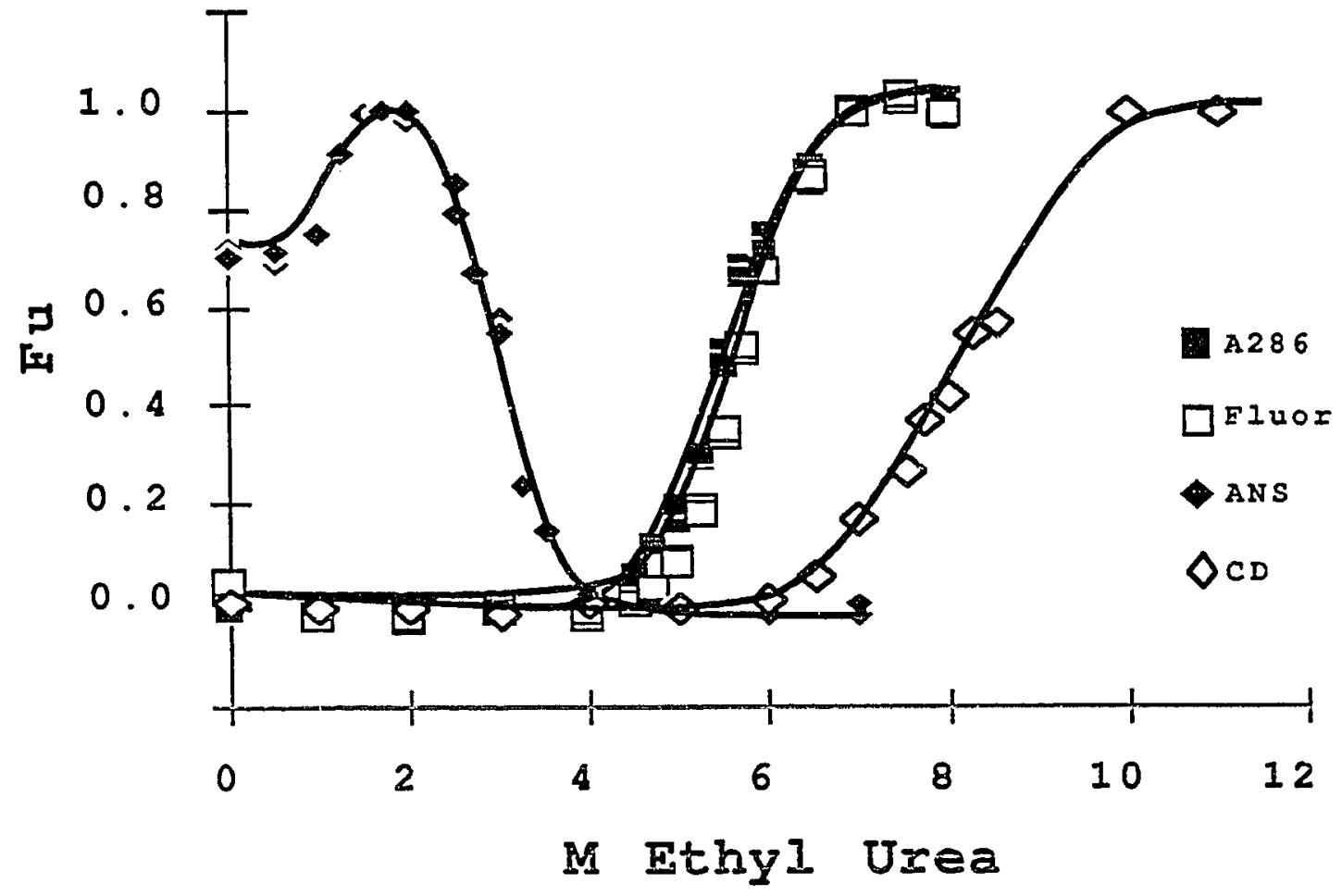

Figure 18. Fraction unfolded of RNase A vs M ethyl urea at $\mathrm{pH} 4.0$ and $17 \pm 1^{\circ} \mathrm{C}$. 
second intermediate, $I_{2}$. Both of these intermediate structures are observed at low denaturant concentrations and have the spectral properties of native protein. The two intermediates are similar in compactness as judged by the unfolding of the urea gradient gels, however, they differ in exposure of hydrophobic groups. The $I_{I}$ intermediate has more hydrophobic pockets on its surface than native protein and these pockets are lost upon formation of $I_{2}$.

The transitions followed by absorbance and fluorescence were found to be coincident with each other, but were not coincident with the circular dichroism data. The absorbance and fluorescence data indicates the presence of third intermediate structure, $I_{3}$, which has absorbance and fluorescence properties of unfolded protein, but has the secondary structure of native protein. Such properties are indicative of a molten globule state. The CD transition occur at higher denaturant concentration than any of the others. This transition indicates the loss of the secondary structure. Since the urea gel transition is coincident with the $C D$ transition, it is reasonable that the decomposition of $I_{3}$ results in the formation of completely unfolded protein, $\mathrm{U}$.

From the above interpretation, the following scheme is proposed for the unfolding process in urea, ethylurea, Nmethylurea, formamide; 1,3-dimethylurea: and acetamide:

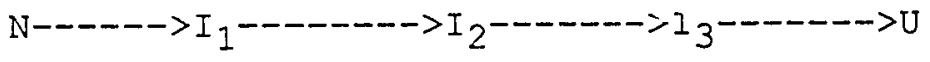

In the case of GuHCl, the observed transitions were very cooperative (Figure 19). The transitions observed by absorbance, fluorescence and circular dichroism were all coincident with each other, indicating a cooperative disruption of the entire protein structure without the population of any intermediate structure. ANS binding studies could not be done with GuHCl, because ANS formed a precipitate with GuHCl, thereby decreasing the solubility of 


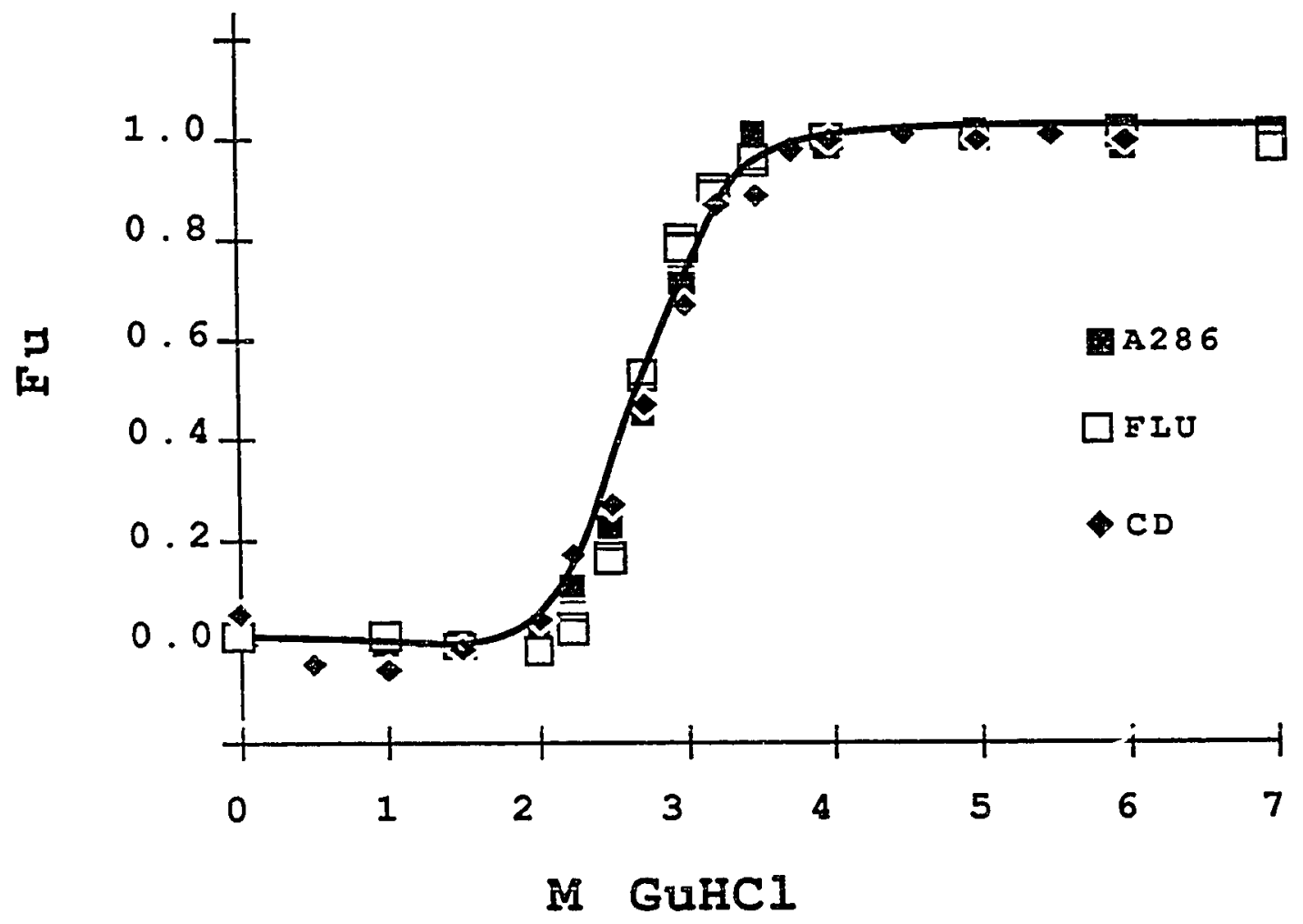

Figure 19. Fraction unfolded of RNase A vs molar GuHCI at $\mathrm{pH} 4.0$ and $17 \pm 1^{\circ} \mathrm{C}$. 
the former. The following scheme is proposed for the unfolding process in GuHCl:

$\mathrm{N}-----D->U$

The absorbance and fluorescence data for DMSO could not be obtained due to the high dependence of the molar absorptivity and molar fluorescence of solvent-exposed tyrosine residues on the concentration of DMSO. Figure 20 shows the overlay plot of data obtained from hydrophobic dye binding, DMSO gradient gels, and $C D$. It can be clearly seen from the plot that ANS and the first gel transition are relatively coincident. This suggests the presence of an intermediate structure, $I_{1}$, produced by partial disruption of the tertiary structure without an effect on the secondary structure. The ANS 2 transition occurs within the "plateau" region of the gel data. This result indicate the formation of a second intermediate, $I_{2}$, which is as compact as $I_{1}$, but differs in the exposure of hydrophobic residues.

The $C D$ and the final gel transition occur in approximately the same region. The coincidence or noncoincidence cannot be determined precisely, as the $C D$ transition is not complete. Assuming that they are coincident, the transition indicates the disruption of the secondary and residual tertiary structure. The working model for the unfolding of RNase $A$ in DMSO is as follows:

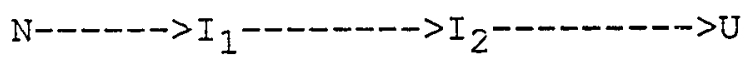

\subsection{CONCLUSION}

The effect of different denaturants on the denaturantinduced unfolding transition was studied by absorbance, fluorescence, circular dichroism, hydrophobic dye binding and by denaturant-gradient gel electrophoresis at $\mathrm{pH} 4.0$.

The denaturants used in this study can be divided into the following categories: denaturants which differ in the 


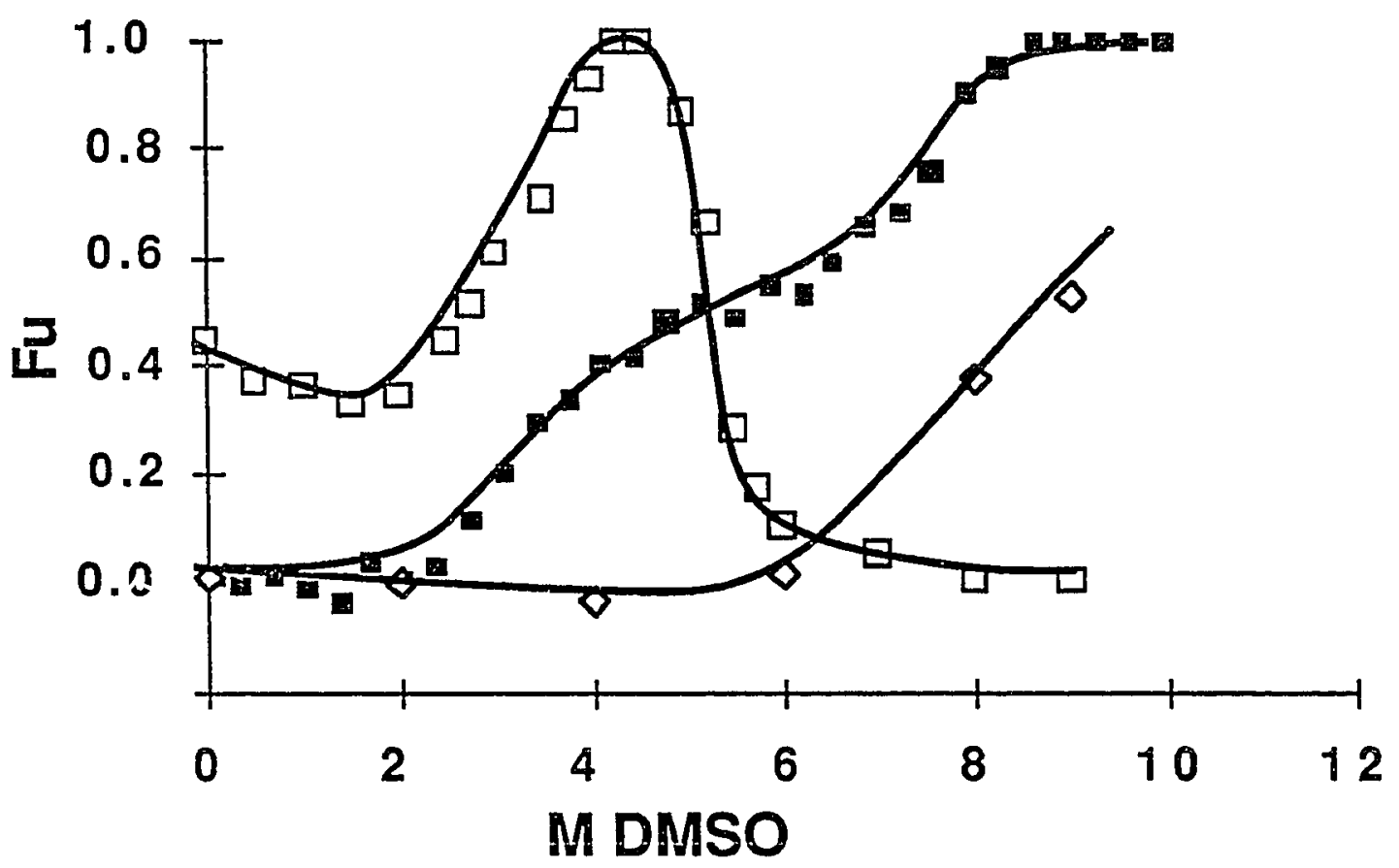

Figure 20. Fraction unfolded of RNase A vs M DMSO as monitored by Hydrophobic dye binding data ( $\square$ ) Diso gradient gel data( $)$, and circular dichroism data( $(0)$ at $\mathrm{pH} 4.0$ and $17 \pm 1^{\circ} \mathrm{C}$. 
number of hydrogens available for hydrogen bonding (reduced ability to act as hydrogen bond donors), the denaturants which differ in the number of nitrogens (reduced ability to act as hydrogen bond acceptors), and the hydrophobic denaturants. The unfolding pathway induced by DMSO differs so markedly from the rest, it is discussed in a separate section.

Reduction in the ability of a denaturant to act as either a hydrogen bond acceptor or a hydrogen bond donor results in an increase in the $\mathrm{Cm}$ for the transition, the former having a greater affect than the latter. From the data observed by absorbance, fluorescence and circular dichroism, the denaturants used can be categorized in the order of decreasing strength as follows:

GuHCl > urea > ethylurea > N-methylurea > 1,3-dimethylurea > formamide > acetamide

Transitions observed for urea and urea derivatives were less cooperative than the others indicating that intermediate states can be populated with these denaturants. In addition, cooperativity decreases with decreasing denaturant strengti which indicates that the stability of intermediate states is enhanced in the presence of weaker denaturants within this class. Eurthermore, the data from the Asis transitions indicates that the hydrogen bonds which are disrupted at low denaturant concentrations are probably involved in tertiary structure interactions rather than in the secondary structure and the hydrophobicity of the urea derivatives is a major factor in the observed transitions.

For all denaturants except GuHCl and DMSO, the ANS and CD transitions were not coincident with each other or the mutually coincident Eluorescence and absorbance transitions. The ron-coincicence indicates the presence of stabie and highly populated intermediate states; three stabiiized intermediate states were observed with each of these 
denaturants. On the other hand, GuHCl showed a two-state transition suggesting that the entire protein structure is disrupted cooperatively.

DMSO apparently unfolds the protein via a different pathway than the other denaturants. DMSO apparently disrupts the hydrophobic core prior to the disruption of the secondary structures. Further studies with more hydrophobic denaturants are required to investigate the protein folding pathway induced by the hydrophobic denaturants. 


\section{References}

Ahmad, F. and Bigelow, C.C. (1982) J. Biol. Chem. 257, 12935. Anfinsen, C.B., Sela, M. and White, F.H. (1957) Science 125, 691.

Baumann, G., Frommel, C., \& Sander, C. (1989) Protein Eng. 2, 329.

Biringer, R.G. and Fink, A.I. (1982a) Biochemistry 21, 4748. Biringer, R.G. \& Fink, A.I. (1982b) J. Mol. Biol. 160, 87 116.

Biringer, R.G. \& Fink, A.L. (1988) Biochemistry 27, 315.

Bowie, J.U., Reidhaar-olsen, J. F., Iim, W.A., \& Sauer, R.T. (1990) Science 247, 1306.

Brand, I. and Bernard, W. (1967) Methods in Enzymology 11, 776.

Brandts, J.F, Halvorson, H.R., Brenon, M. (1975) Biochemistry 14,4953 .

Chothia, C. (1976) J. MOI. Biol. 105, 1.

Creighton, T.E., (1990) Biochem. J. 270, 1.

Carlsberg, Ser. Chim. 30, 21.

Cassim, J.Y., and Yang, J.T. (1969) Biochemistry 8, 194.

Chen, G.C., and Yang, J.T. (1977) Anal. Lett. 10, 1195.

Creighton, T.E. (1986) Methods in Enzymology 131, 156.

Edelboch, H., \& Osborne, J.C. (1976) Adv. Protein Chem. 30, 183.

Garel, J.R., Baldwin, R.I. (1973) Proc. NatI. Acad. Sci. USA 70,3347 .

Green, R.F. and Pace, C.N. (1974) J. Biol. Chem. 249, 5388. Harrington, W. F., and Schellman, J. A. (1956) Compt. Rend.

Trav. Lab. Carlsberg, Ser. Chim. 30, 21.

Hibbard, L.S., \& Tulinsky, A. (1978) Biochemistry 17, 5460.

Johnson, W.C. Jr., (1990) Protein 7, 205-214.

Karplus, M., Weaver, D.I. (1976) Nature 260, 404.

Kauzmann, W. (1954) in The Mechanism of Enzyme Action, (W.B. MCElroy and B. Glass, Eds.), The John Hopkins

Press, Baltimore. 
Kauzmann, W. (1959) Adv. Protein Chem. 14, 1.

Kendrew, J.C., Dickerson, R.E., Strandberg, B.E., Hart, R.G.,

Davies, D. R., Phillips, D.C., and Shore, V.C. (1960)

Nature, 185, 422 .

Kim, P. S., Baldwin, R., I., (1980) Biochemistry 19, 6124.

LaPorte, D.C., Wierman, B.M., and Storm, D.R. (1980)

Biochemistry 16, 3814 .

Johnson, W.C. Jr., (1990) Proteins 7, 205.

Levinthal, C. (1968) J. Chim. Phys. 65:, 44.

Levitt, M., Warshel, A. (1975) Nature 253, 694.

Iinderstrom-Lang, K.U. (1952) in Lane Medical Lectures,

Vol.6, Stanford University Press, Stanford, CA.

Lupu-Lotan, N., Yaron, A., Berger, A., \& Sela. M. (1965)

Biopolymers 3, 625.

Matheson, R.R.; Jr \& Scheraga, H.A. (1979) . Biochemistry. 18, 2437.

Mc Wherter, et al. (1384) Anal. Biochem. 141, 523.

Northrop, J.H. (1932) J. Gen. Physiol. 16, 323.

Pace, N.C. (1986) Methods in Enzymology 131, 266.

Pace, C., N. (1975) CRC Crit. Rev. Biochem. 3, 1.

Pauling, I., Corey, R.B., \& Branson, H.R. (1951) Proc. Natl.

Acad. SCi. U.S.A. 37, 205.

Ptitsyn, O.B., Rashin, A.A. (1975) Biophys. Chem. 3: 1.

Ptitsyn, O.B., Finkelstein, A.V. (1980) Q. Rev. Biophys. 13, 339.

Ptitsyn, O.B. (1987) J. Protein Chem. 6, 273.

Regan, L., \& DeGrado, W.F. (1988) Science 241, 976.

Richarson, J.S., and Richarson, D.C., (1990) in Protein

Folding, (I.M.Gierasch and J. King, Ed.) p 5, American

Association for the Advancement of Science, Washington.

Roseman, D., \& Jencks, W.P. (1975) J. Am. Chem. Soc. 97,

631.

Schellman, J.A. (1978) Biopolymers 17, 1305.51.

Schmid, F.X. \& Baldwin, R.L. (1979), J. Mol. Biol. 135, 199.

Schmid, F.X. (1986) Methods of Enzymology 131, 70.

Singer, S.J. (1962) Adv. Proteir Chem. 17, 1. 
Stigter, D., \& Dill, K.A. (1990) Biochemistry 29, 1262.

Tanford, C. (1968). Adv. Protein Chem. 23, 121.

von Hippel, P. H. \& Schleich, T. (1969) ACC. Chem. Res. 2, 257.

Vinogradov, S.N. \& Iinnell, R.H. (1971) in Hydrogen Bonding, Van Nostrand Reinhold, New York.

Wagner, G. (1983) Q. Rev. Biophys. 16, 1 .

Wertz, D.H., \& Scheraga, H. A. (1978) Macromolecules 11, 9. Wetlaufer, D.B. (1973) Proc. Natl. Acad. Sci. U.S. A. 70, 697.

Wu, H. (1929) Am. J. Physiol. 90, 562. 